\title{
Convergence Rates to Stationary Solutions of a Gas-liquid Model with External Forces and Vacuum
}

\author{
Long Fan Qingling LiU ChangJiang ZhU* \\ The Hubei Key Laboratory of Mathematical Physics \\ School of Mathematics and Statistics \\ Central China Normal University, Wuhan 430079, P.R. China
}

\begin{abstract}
In this paper, we study the asymptotic behavior of solutions to a Gas-liquid model with external forces and general pressure law. Under some suitable assumptions on the initial date and $\gamma>1$, if $\theta \in\left(0, \frac{\gamma}{2}\right] \cap(0, \gamma-1] \cap(0,1-\alpha \gamma]$, we prove the weak solution $(c Q(x, t), u(x, t))$ behavior asymptotically to the stationary one by adapting and modifying the technique of weighted estimates. In addition, if $\theta \in\left(0, \frac{\gamma}{2}\right] \cap(0, \gamma-1) \cap(0,1-\alpha \gamma]$, following the same idea in [8], we estimate the stabilization rate of the solution as time tends to infinity in the sense of $L^{\infty}$ norm.
\end{abstract}

Key Words: Gas-liquid model, stationary solutions, vacuum, convergence rates.

Mathematics subject classifications (2000): 76T10, 35L65, 35B40.

\section{Contents}

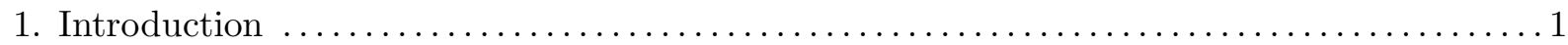

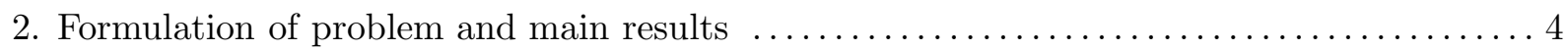

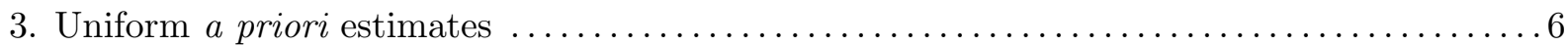

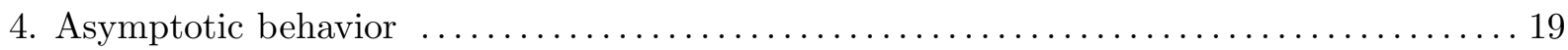

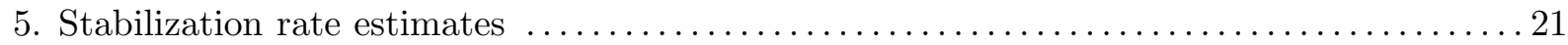

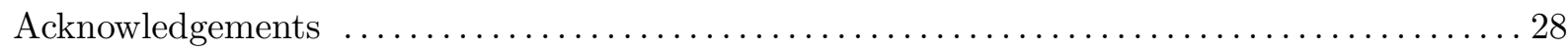

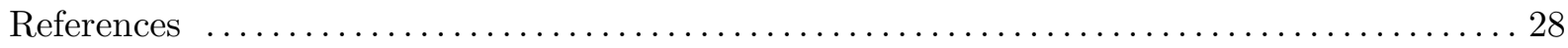

\section{Introduction}

The one-dimensional two-phase model of the drift-flux type is frequently used to simulate unsteady, compressible flow of liquid and gas in pipes and wells (cf. 1, 4]). The model consists of two mass conservation equations corresponding to each of the two phases gas $(g)$ and liquid $(l)$

\footnotetext{
*Corresponding author. Email: cjzhu@mail.ccnu.edu.cn
} 
and one equation for the momentum of the mixture and takes the following form

$$
\left\{\begin{array}{l}
\partial_{t}\left[\alpha_{g} \rho_{g}\right]+\partial_{x}\left[\alpha_{g} \rho_{g} u_{g}\right]=0 \\
\partial_{t}\left[\alpha_{l} \rho_{l}\right]+\partial_{x}\left[\alpha_{l} \rho_{l} u_{l}\right]=0 \\
\partial_{t}\left[\alpha_{g} \rho_{g} u_{g}+\alpha_{l} \rho_{l} u_{l}\right]+\partial_{x}\left[\alpha_{g} \rho_{g} u_{g}^{2}+\alpha_{l} \rho_{l} u_{l}^{2}+p\right]=-q+\partial_{x}\left[\varepsilon \partial_{x} u_{m i x}\right]
\end{array}\right.
$$

where $u_{m i x}=\alpha_{g} u_{g}+\alpha_{l} u_{l}$ and the unknown variables $\alpha_{g}, \alpha_{l} \in[0,1]$ denote volume fractions satisfying the fundamental relation:

$$
\alpha_{g}+\alpha_{l}=1
$$

Furthermore, the other unknown variables $\rho_{g}, \rho_{l}, u_{g}, u_{l}$ denote gas density, liquid density, velocities of gas and liquid respectively, whereas $p$ is the common pressure for both phases, $q$ presents external forces, like gravity and friction, and $\varepsilon>0$ denotes viscosity.

As in [5], we assume the liquid is incompressible, and the gas is polytropic, i.e.,

$$
\rho_{l}=\text { constant }, \quad p=A \rho_{g}^{\gamma}, \quad \gamma>1, \quad A>0 .
$$

For the relation between the drift-flux model and the more general two-fluid and the simplification of the model, one can refer to [6] and [5] for more details. Here, we have skipped them and we directly study the following gas-liquid model described in terms of Lagrangian variables (cf. [5]) with frictional force term $-f m^{2} u|u|(f \geq 0)$ and gravity term $g(>0)$ :

$$
\left\{\begin{array}{l}
n_{t}+(n m) u_{x}=0 \\
m_{t}+m^{2} u_{x}=0, \\
u_{t}+p(n, m)_{x}=-f m^{2} u|u|+g+\left(\varepsilon(m, n) m u_{x}\right)_{x}, \quad 0<x<1, \quad t>0,
\end{array}\right.
$$

with

$$
p(n, m)=A\left(\frac{n}{\rho_{l}-m}\right)^{\gamma}, \quad \varepsilon(m, n)=\frac{B n^{\theta}}{\left(\rho_{l}-m\right)^{\theta+1}}, \quad A, B>0 .
$$

Boundary conditions are given by

$$
n(0, t)=m(0, t)=0, \quad u(1, t)=0, \quad t \geq 0,
$$

whereas initial data are

$$
n(x, 0)=n_{0}(x), m(x, 0)=m_{0}(x), u(x, 0)=u_{0}(x), x \in[0,1] .
$$

Without loss of generality, we take $A=B=1$.

Let's first review some of the previous works in this direction. For the simplified model obtained by neglecting frictional force and gravity in (1.3), there has been a large number of research results when the viscosity function $\varepsilon(m, n)$ takes different forms. Clearly speaking, Evje and Karlsen in [3] studied the existence of the global weak solutions when the initial masses connected to vacuum discontinuously for the viscosity function $\varepsilon(m, n)$ taking the following form

$$
\varepsilon(m)=\frac{m^{\theta}}{\left(\rho_{l}-m\right)^{\theta+1}}, \quad \theta \in\left(0, \frac{1}{3}\right)
$$


This result was later improved to the case $\theta \in(0,1]$ by Yao and Zhu in [20]. When the initial masses connected to vacuum continuously, Evje, Flatten and Friis in [7] also obtained the global existence and uniqueness of weak solutions for the viscosity $\varepsilon(m, n)$ taking the following form

$$
\varepsilon(n, m)=\frac{n^{\theta}}{\left(\rho_{l}-m\right)^{\theta+1}}, \quad \theta \in\left(0, \frac{1}{3}\right) .
$$

When the viscosity is constant and the initial masses connected to vacuum continuously, the existence of global weak solutions was obtained by Yao and Zhu in [21]. Recently, Friis and Evje in [5] proved the existence of global weak solutions to the initial boundary value problem of (1.3) with initial data (1.6) and boundary conditions $\left[p(n, m)-\varepsilon(m, n) m u_{x}\right](0, t)=0, u(1, t)=0$ when the viscosity $\varepsilon(m, m)$ takes the form (1.7).

However, there are few results on the asymptotic behavior and decay rate estimates for the initial boundary value problem (1.3)-(1.6). In this paper, we rewrite our problem into (2.2)(2.4) similar to the model of $1 \mathrm{D}$ compressible Navier-Stokes equations with gravity by using the variable transformations as in [3, 7] and study the asymptotic behavior and decay rate of (2.2)-(2.4) by similar line as in [2, 8, 26]. According to the method in [26], we know that the uniform upper and lower bounds of $c Q$ in (2.2) and the uniform bound of

$$
\int_{0}^{t} \int_{0}^{1} c^{\theta} Q^{1+\theta} u_{x t}^{2} d x d s
$$

play a very important role in studying the asymptotic behavior of $c Q$ and $u$. In order to obtain these uniform bounds, we have to deal with the difficulties which come from the additional external force $-h(Q) u|u|$ in (2.2) which is more complex than the only constant external force $g$ in [26]. To overcome this difficulty, we use a priori assumption in the proof of the uniform upper and lower bounds of $c Q$. Furthermore, we obtain the higher regularity of the velocity function $u$ by using a new skill. Clearly speaking, we get the uniform estimate of $\|u(\cdot, t)\|_{L^{p}([0,1])}$ $(p=2,4,5)$ and $\|u\|_{L^{q}([0,1] \times[0, \infty))}(q=2,3,4)$ with the help of the recurrence method. The higher regularity of the velocity function $u$ improves the estimate of $\|u\|_{L^{2}([0,1] \times[0, \infty))}$ in the previous works [2, 8, 26]. Based on these estimates, we get the uniform bound of (1.9).

It is necessary for us to illustrate that the main methods used to obtain our results are similar to those in [2, 8, 26]. In view of this, let's review some of the relevant works about Navier-Stokes equations with density-dependent viscosity and vacuum. For the case without external force, Guo and Zhu in [11, 12] gave the asymptotic behavior and decay rate of the density function $\rho(x, t)$ when the initial density connects to vacuum continuously. Zhu in [25] investigated the asymptotic behavior and decay rate estimates on the density function $\rho(x, t)$ by overcoming some new difficulties which came from the appearance of boundary layers when the initial density connects to vacuum discontinuously. In [11, 12, 25], the auxiliary function $w(x, t)$ introduced by Nagasawa in [14] was used to investigate the decay rate of $\rho(x, t)$. For the other case with gravity, under some assumptions on the initial data, Zhang and Fang in [8] proved that the solution converges to the stationary states as time goes to infinity provided $\theta \in(0, \gamma-1) \cap\left(0, \frac{\gamma}{2}\right]$ and $\gamma>1$. The stabilization rates were also estimated in several norms. Duan in [2] generalized part result in [8], and showed that the solution converges to the stationary state in the sense of integral when $\gamma=2, \theta=1$. Recently, Zhu and $\mathrm{Zi}$ in [26] improved the results in [2, 8] in the sense that $\theta \in(0, \gamma-1] \cap\left(0, \frac{\gamma}{2}\right]$.

The rest of this paper is organized as follows. In Section 2, the system is translated into a more simple one, then we give the definition of the weak solution and state the main results. 
In Section 3, we derive some crucial uniform estimates for studying the asymptotic behavior and the decay rate estimates. In Section 4, the asymptotic behavior of weak solution will be given. In Section 5, we will establish stabilization rate estimates of the solution as time tends to infinity.

\section{Formulation of problem and main results}

In this section, we rewrite our problem (1.3)-(1.6) into (2.2)-(2.4) by using the same transformations as in [3, 7], then we state our main results in Theorem 2.3 and Theorem 2.4.

Firstly we introduce the transformations

$$
c=\frac{n}{m}, \quad Q(m)=\frac{m}{\rho_{l}-m} .
$$

Form the first two equations of (1.3), we get

$$
c_{t}=\frac{n_{t}}{m}-\frac{n}{m^{2}} m_{t}=-\frac{m n u_{x}}{m}+\frac{n m^{2}}{m^{2}} u_{x}=0,
$$

and

$$
\begin{aligned}
Q(m)_{t}=\left(\frac{m}{\rho_{l}-m}\right)_{t} & =\left(\frac{1}{\rho_{l}-m}+\frac{m}{\left(\rho_{l}-m\right)^{2}}\right) m_{t} \\
& =\frac{\rho_{l}}{\left(\rho_{l}-m\right)^{2}} m_{t}=-\frac{\rho_{l} m^{2}}{\left(\rho_{l}-m\right)^{2}} u_{x} \\
& =-\rho_{l} Q(m)^{2} u_{x} .
\end{aligned}
$$

Then we can rewrite the initial boundary problems (1.3)-(1.6) into the following forms:

$$
\left\{\begin{array}{l}
c_{t}=0 \\
Q_{t}+\rho_{l} Q^{2} u_{x}=0, \\
u_{t}+(c Q)_{x}^{\gamma}=-h(Q) u|u|+g+\left(c^{\theta} Q^{1+\theta} u_{x}\right)_{x}, \quad 0<x<1, \quad t>0,
\end{array}\right.
$$

with initial data

$$
(c Q, u)(x, 0)=\left(c_{0} Q_{0}, u_{0}\right)(x), \quad 0 \leq x \leq 1,
$$

and the boundary conditions

$$
(c Q)(0, t)=0, \quad u(1, t)=0, \quad t>0 .
$$

Here

$$
h(Q)=f \rho_{l}^{2} \frac{Q^{2}}{(1+Q)^{2}}, \quad f \geq 0
$$

and $c_{0}, Q_{0}$ is given from $\left[n_{0}, m_{0}\right]$ according the transformations (2.1). In particular, the first equation of (2.2) implies that $c(x, t)=c_{0}(x):=\frac{n_{0}}{m_{0}}(x)$.

Let $\left(c Q_{\infty}\right)(x)$ be the solution of the following stationary problem:

$$
\left\{\begin{array}{l}
\left(c Q_{\infty}\right)_{x}^{\gamma}=g \\
\left(c Q_{\infty}\right)(0)=0
\end{array}\right.
$$


Then

$$
\left(c Q_{\infty}\right)(x)=(g x)^{\frac{1}{\gamma}} .
$$

Throughout this paper, our assumptions on the initial data $c_{0}, Q_{0}, u_{0}$ are as follows:

$\left(A_{1}\right) \quad C_{1} x^{\frac{1}{\gamma}} \leq c_{0} Q_{0}(x) \leq C_{2} x^{\frac{1}{\gamma}}, C_{3} x^{\alpha} \leq c_{0}(x) \leq C_{4} x^{\alpha}$, with some positive constants $0<C_{1} \leq C_{2}, 0<C_{3} \leq C_{4}, 0 \leq \alpha<\frac{1}{\gamma}$;

$\left(A_{2}\right) \quad u_{0} \in H^{1}([0,1]) \cap L^{5}([0,1]), \int_{0}^{1} x^{\frac{(2+\alpha) \gamma-\theta-1}{\gamma}}\left(\left(c Q_{0}\right)_{x}^{\theta}-\left(c Q_{\infty}\right)_{x}^{\theta}\right)^{2} d x \leq C ;$

$\left(A_{3}\right) \quad\left(c^{\theta} Q_{0}^{1+\theta} u_{0 x}\right)_{x} \in L^{2}([0,1])$.

Under assumptions $\left(A_{1}\right)-\left(A_{3}\right)$, we will study the asymptotic behavior of $c Q$ and $u$ provided that the global weak solution to the initial boundary value problem (2.2)-(2.4) exists. In the Lagrangian coordinates, the definition of the weak solution to (2.2)-(2.4) can be stated as follows:

Definition 2.1. (Weak solution) A pair of functions $(c Q(x, t), u(x, t))$ is called a weak solution to the initial boundary problem (2.2) $-(2.4)$, if

$$
\begin{gathered}
c \in C^{1}([0,1] \times[0, \infty)), \quad c Q, u \in L^{\infty}([0,1] \times[0, \infty)) \cap C^{1}\left([0, \infty) ; L^{2}(0,1)\right), \\
c^{\theta} Q^{1+\theta} u_{x} \in L^{\infty}([0,1] \times[0, \infty)) \cap C^{\frac{1}{2}}\left([0, \infty) ; L^{2}(0,1)\right) .
\end{gathered}
$$

Furthermore, the following equations hold:

$$
Q_{t}+\rho_{l} Q^{2} u_{x}=0, \text { a.e., }
$$

and

$$
\int_{0}^{\infty} \int_{0}^{1}\left\{u \phi_{t}+\left((c Q)^{\gamma}-c^{\theta} Q^{1+\theta} u_{x}\right) \phi_{x}+g \phi-h(Q) u|u| \phi\right\} d x d t+\int_{0}^{1} u_{0}(x) \phi(x, 0) d x=0,
$$

for any test functions $\phi(x, t) \in C_{0}^{\infty}(\Omega)$ with $\Omega=\{(x, t): 0 \leq x \leq 1, t \geq 0\}$.

Remark 2.2. (Existence of the global weak solution). To our knowledge, by using the standard line method (see [5, 15] for example), it is easy to obtain the global existence of the weak solutions to (2.2)-(2.4). The details are omitted.

In what follows, $C$ denotes a generic positive constant depending on initial data, $\gamma$ and $\theta$, etc., but independent of $t . \delta, \epsilon_{0}$ denote some positive (generally small) constants.

The main results in this paper can be stated as follows:

Theorem 2.3. Let $c_{0}, Q_{0}, u_{0}$ satisfy $\left(A_{1}\right)-\left(A_{3}\right), \gamma>1$ and $\theta \in\left(0, \frac{\gamma}{2}\right] \cap(0, \gamma-1] \cap(0,1-\alpha \gamma]$. There exists a constant $0<\epsilon_{0}<1$, such that if

$$
\left\|u_{0}\right\|_{L^{2}}^{2} \leq \epsilon_{0}, \quad \int_{0}^{1} x^{1-3 / \gamma}\left(c Q_{0}-c Q_{\infty}\right)^{2} d x \leq \epsilon_{0}
$$

the weak solution $((c Q)(x, t), u(x, t))$ to the initial boundary value problem (2.2)-(2.4) satisfies

$$
\lim _{t \rightarrow \infty}(c Q)(x, t)=\left(c Q_{\infty}\right)(x)
$$


uniformly in $x \in[0,1]$ and

$$
\lim _{t \rightarrow \infty} \sup _{x \in[\delta, 1]}|u(x, t)|=0,
$$

for any $0<\delta<1$. Furthermore, if we assume further $\theta<\gamma-1$, then (2.11) can be improved as

$$
\lim _{t \rightarrow \infty} \sup _{x \in[0,1]}|u(x, t)|=0 .
$$

Theorem 2.4. Under the conditions of Theorem 2.3 and $\theta<\gamma-1$, we have

$$
\left\|\left(c Q^{\theta}-c Q_{\infty}^{\theta}\right)(\cdot, t)\right\|_{L^{\infty}} \leq C(1+t)^{-\frac{2 \theta}{4 \gamma+\alpha \gamma-2}},
$$

and

$$
\|u(\cdot, t)\|_{L^{\infty}([0,1])} \leq C(1+t)^{-\frac{1}{2}}
$$

for all $t \geq 0$.

Remark 2.3. If the coefficient $f=0$ of the frictional force term in (2.2) and $\alpha=0, C_{3}=C_{4}$ in the assumption $\left(A_{1}\right)$, then (2.2) is simplified into Navier-Stokes equations with gravity. In this case, we need not deal with the additional external force $-h(Q) u|u|$ in (3.9) and derive $\theta \in\left(0, \frac{\gamma}{2}\right] \cap(0, \gamma-1]$. Compared with the results in $[2,8$, 26], our results can be viewed as a generalization of the ones in $[2,8,26]$.

\section{Uniform a priori estimates}

In this section, we will derive some uniform-in-time a priori estimates for the solutions to (2.2)(2.4) by classical energy method. First, we list some elementary equalities which follow from (2.2) directly. These equalities will be used frequently later.

Lemma 3.1 Under the conditions of Theorem 2.3, it holds that for $0<x<1, t>0$,

$$
\begin{gathered}
\left((c Q)^{\theta}\right)_{t}=\theta \rho_{l}\left(c Q_{\infty}\right)^{\gamma}-\theta \rho_{l}(c Q)^{\gamma}-\theta \rho_{l} \int_{0}^{x} u_{t} d y-\theta \rho_{l} \int_{0}^{x} h(Q) u|u| d y \\
(c Q)^{\gamma}-\left(c Q_{\infty}\right)^{\gamma}=-\frac{1}{\theta \rho_{l}}\left((c Q)^{\theta}\right)_{t}-\int_{0}^{x} u_{t} d y-\int_{0}^{x} h(Q) u|u| d y
\end{gathered}
$$

and

$$
\left((c Q)^{\theta}-\left(c Q_{\infty}\right)^{\theta}\right)_{x t}=-\theta \rho_{l}\left((c Q)^{\gamma}-\left(c Q_{\infty}\right)^{\gamma}\right)_{x}-\theta \rho_{l} u_{t}-\theta \rho_{l} h(Q) u|u| .
$$

Proof. The above three equalities follow from (2.2) and (2.4) directly.

The basic energy estimate similar to [2] for the case $\theta=1, \gamma=2$ and [8] is given as follows:

Lemma 3.2. (Basic energy estimate). Under the conditions of Theorem 2.3, it holds that

$$
\begin{aligned}
& \int_{0}^{1}\left(\frac{1}{2} u^{2}+\frac{1}{\rho_{l}} \int_{Q_{\infty}}^{Q} \frac{c^{\gamma}\left(h^{\gamma}-Q_{\infty}^{\gamma}\right)}{h^{2}} d h\right) d x+\int_{0}^{t} \int_{0}^{1} c^{\theta} Q^{1+\theta} u_{x}^{2} d x d s \\
& +\int_{0}^{t} \int_{0}^{1} h(Q) u^{2}|u| d x d s \leq C \epsilon_{0}, \quad 0<t<\infty,
\end{aligned}
$$

where $C$ is a positive constant, independent of $t$ and $\epsilon_{0}$. 
Proof. Multiplying (2.2) 2 and (2.2) $)_{3}$ by $\frac{c^{\gamma}\left(Q^{\gamma}-Q_{\infty}^{\gamma}\right)}{Q^{2}}$ and $u$ respectively, and integrating the result equations with respect to $x$ and $t$ over $[0,1]$ and $[0, t]$, by using the boundary conditions (2.4), we have

$$
\begin{aligned}
& \int_{0}^{1}\left(\frac{1}{2} u^{2}+\frac{1}{\rho_{l}} \int_{Q_{\infty}}^{Q} \frac{c^{\gamma}\left(h^{\gamma}-Q_{\infty}^{\gamma}\right)}{h^{2}} d h\right) d x+\int_{0}^{t} \int_{0}^{1} h(Q) u^{2}|u| d x d s+\int_{0}^{t} \int_{0}^{1} c^{\theta} Q^{1+\theta} u_{x}^{2} d x d s \\
= & \int_{0}^{1}\left(\frac{1}{2} u_{0}^{2}+\frac{1}{\rho_{l}} \int_{Q_{\infty}}^{Q_{0}} \frac{c^{\gamma}\left(h^{\gamma}-Q_{\infty}^{\gamma}\right)}{h^{2}} d h\right) d x .
\end{aligned}
$$

It follows from $\left(A_{1}\right)$ and (2.6) that

$$
\begin{aligned}
\int_{0}^{1} \int_{Q_{\infty}}^{Q_{0}} \frac{c^{\gamma}\left(h^{\gamma}-Q_{\infty}^{\gamma}\right)}{h^{2}} d h d x & \leq C \int_{0}^{1} Q_{\infty}^{-2}\left|Q_{0}-Q_{\infty}\right|\left|\left(c Q_{0}\right)^{\gamma}-\left(c Q_{\infty}\right)^{\gamma}\right| d x \\
& \leq C \int_{0}^{1}\left(c Q_{\infty}\right)^{-2} \gamma \xi^{\gamma-1}\left|c Q_{0}-c Q_{\infty}\right|^{2} d x \\
& \leq C \int_{0}^{1} x^{1-3 / \gamma}\left(c Q_{0}-c Q_{\infty}\right)^{2} d x
\end{aligned}
$$

where $\xi$ is between $c Q_{0}$ and $c Q_{\infty}$, hence we obtain

$$
\begin{aligned}
& \int_{0}^{1}\left(\frac{1}{2} u^{2}+\frac{1}{\rho_{l}} \int_{Q_{\infty}}^{Q} \frac{c^{\gamma}\left(h^{\gamma}-Q_{\infty}^{\gamma}\right)}{h^{2}} d h\right) d x+\int_{0}^{t} \int_{0}^{1} c^{\theta} Q^{1+\theta} u_{x}^{2} d x d s \\
& +\int_{0}^{t} \int_{0}^{1} h(Q) u^{2}|u| d x d s \leq C \epsilon_{0} .
\end{aligned}
$$

This completes the proof.

Combining the the smallness of $\sup _{t \geq 0}\|u(\cdot, t)\|_{L^{2}(0,1)}$ obtained by the basic energy estimate and a priori assumption, we could get the uniform upper and lower bounds for $Q(x, t)$ which will play a crucial role in studying the asymptotic behavior of $c Q$ and $u$.

Lemma 3.3. Under the conditions in Theorem 2.3, we have

$$
K_{1} Q_{\infty}(x) \leq Q(x, t) \leq K_{2} Q_{\infty}(x)
$$

where $(x, t) \in \Omega=\{(x, t): 0 \leq x \leq 1, t \geq 0\}, K_{1}$ and $K_{2}$ are two positive constants, independent of $t$.

Proof. Let $Y(x, t)=(c Q)^{\theta}(x, t)\left(c Q_{\infty}\right)^{-\theta}(x)$, then due to (3.1), we have

$$
Y_{t}=\theta \rho_{l}\left(c Q_{\infty}\right)^{\gamma-\theta}\left(1-Y^{\frac{\gamma}{\theta}}\right)-\theta \rho_{l}\left(\left(c Q_{\infty}\right)^{-\theta} \int_{0}^{x} u d y\right)_{t}-\theta \rho_{l}\left(c Q_{\infty}\right)^{-\theta} \int_{0}^{x} h(Q) u|u| d y
$$

Assume

$$
Y \leq C_{5}+1
$$


where $C_{5}=g^{-\frac{\theta}{\gamma}} C_{2}^{\theta}$. The last term on the right side of (3.7) can be estimated as follows

$$
\begin{aligned}
-\theta \rho_{l}\left(c Q_{\infty}\right)^{-\theta} \int_{0}^{x} h(Q) u|u| d y & \leq C \delta\left(c Q_{\infty}\right)^{-3 \theta} \int_{0}^{x} h(Q) d y+C(\delta) \int_{0}^{x} h(Q) u^{2}|u| d y \\
& \leq C \delta\left(c Q_{\infty}\right)^{-3 \theta} \int_{0}^{x} Q^{2} d y+C(\delta) \int_{0}^{x} h(Q) u^{2}|u| d y \\
& \leq C \delta\left(c Q_{\infty}\right)^{-3 \theta} \int_{0}^{x}\left(Q^{2} Q_{\infty}^{-2}\right) Q_{\infty}^{2} d y+C(\delta) \int_{0}^{x} h(Q) u^{2}|u| d y \\
& \leq C \delta\left(c Q_{\infty}\right)^{-3 \theta}\left[\max Y^{\frac{2}{\theta}}\right] x^{\frac{2}{\gamma}-2 \alpha+1}+C(\delta) \int_{0}^{x} h(Q) u^{2}|u| d y \\
& \leq C \delta\left(C_{5}+1\right)^{\frac{2}{\theta}}\left(c Q_{\infty}\right)^{\gamma-\theta}\left(c Q_{\infty}\right)^{-\gamma-2 \theta} x^{\frac{2}{\gamma}-2 \alpha+1}+C(\delta) \int_{0}^{1} h(Q) u^{2}|u| d y \\
& \leq C \delta\left(C_{5}+1\right)^{\frac{2}{\theta}}\left(c Q_{\infty}\right)^{\gamma-\theta}+C(\delta) \int_{0}^{1} h(Q) u^{2}|u| d y,
\end{aligned}
$$

where we have used Young inequality, and the fact

$$
\left(c Q_{\infty}\right)^{-\gamma-2 \theta} x^{\frac{2}{\gamma}-2 \alpha+1} \leq C,
$$

which follows from (2.6) and $\theta \leq 1-\alpha \gamma$. Then substituting (3.9) into (3.7), we have

$$
\begin{aligned}
Y_{t} \leq & -\theta \rho_{l}\left(\left(c Q_{\infty}\right)^{-\theta} \int_{0}^{x} u d y\right)_{t}+C(\delta) \int_{0}^{1} h(Q) u^{2}|u| d y \\
& +\theta \rho_{l}\left(c Q_{\infty}\right)^{\gamma-\theta}\left(1-Y^{\frac{\gamma}{\theta}}+C \delta\left(C_{5}+1\right)^{\frac{2}{\theta}}\right) .
\end{aligned}
$$

Integrating the inequality above over $[0, t]$ with respect to $t$, we have

$$
\begin{aligned}
Y \leq & Y_{0}+\theta \rho_{l}\left(c Q_{\infty}\right)^{-\theta} \int_{0}^{x}\left(u_{0}-u\right) d y+C(\delta) \int_{0}^{t} \int_{0}^{1} h(Q) u^{2}|u| d y \\
& +\theta \rho_{l}\left(c Q_{\infty}\right)^{\gamma-\theta} \int_{0}^{t}\left(1-Y^{\frac{\gamma}{\theta}}+C \delta\left(C_{5}+1\right)^{\frac{2}{\theta}}\right) d s \\
\leq & Y_{0}+2 \theta \rho_{l}\left(c Q_{\infty}\right)^{-\theta} x^{\frac{1}{2}} \sup _{t \geq 0}\left(\int_{0}^{1} u^{2} d x\right)^{\frac{1}{2}}+C(\delta) \epsilon_{0}+\theta \rho_{l}\left(c Q_{\infty}\right)^{\gamma-\theta} \int_{0}^{t}\left(1-Y^{\frac{\gamma}{\theta}}+C \delta\left(C_{5}+1\right)^{\frac{2}{\theta}}\right) d s \\
\leq & Y_{0}+C \sup _{t \geq 0}\left(\int_{0}^{1} u^{2} d x\right)^{\frac{1}{2}}+C \epsilon_{0}+\theta \rho_{l}\left(c Q_{\infty}\right)^{\gamma-\theta} \int_{0}^{t}\left(1-Y^{\frac{\gamma}{\theta}}+C \delta\left(C_{5}+1\right)^{\frac{2}{\theta}}\right) d s \\
\leq & Y_{0}+\left(C \epsilon_{0}\right)^{\frac{1}{2}}+\theta \rho_{l}\left(c Q_{\infty}\right)^{\gamma-\theta} \int_{0}^{t}\left(1-Y^{\frac{\gamma}{\theta}}+C \delta\left(C_{5}+1\right)^{\frac{2}{\theta}}\right) d s
\end{aligned}
$$

where we have used the fact

$$
\left(c Q_{\infty}\right)_{\infty}^{-\theta} x^{\frac{1}{2}} \leq C
$$

which follows from (2.6) and the restriction $\gamma \geq 2 \theta$ directly. From (3.10), it is easy to get

$$
Y(x, t) \leq \max \left\{\left(1+C \delta\left(C_{5}+1\right)^{\frac{2}{\theta}}\right)^{\frac{\theta}{\gamma}}, Y_{0}+\left(C \epsilon_{0}\right)^{\frac{1}{2}}\right\}:=K_{2},
$$

where $Y_{0}=\left(c Q_{0}\right)^{\theta}\left(c Q_{\infty}\right)^{-\theta} \leq C_{2}^{\theta} g^{-\frac{\theta}{\gamma}}=C_{5}$.

Now, from (3.8), (3.11) and continuity of $Y(x, t)$, we can show that the priori assumption can be closed provided $\epsilon_{0}$ and $\delta$ sufficiently small. Therefore the upper bound of $Y(x, t)$ is obtained. 
Next, we estimate the lower bound of $Y(x, t)$. Similar to the estimate of the upper bound of $Y(x, t)$, one has

$$
Y(x, t) \geq Y_{0}-\left(C \epsilon_{0}\right)^{\frac{1}{2}}+\theta \rho_{l}\left(c Q_{\infty}\right)^{\gamma-\theta} \int_{0}^{t}\left(1-Y^{\frac{\gamma}{\theta}}-C \delta\right) d s
$$

It is easy to get

$$
Y \geq \min \left\{(1-C \delta)^{\frac{\theta}{\gamma}}, Y_{0}-\left(C \epsilon_{0}\right)^{\frac{1}{2}}\right\}:=K_{1} .
$$

The proof of Lemma 3.4 is complete.

The next lemma will be used for many times later, and it has been proved by Duan in [2] for the case $\theta=1, \gamma=2$.

Lemma 3.4. Under the conditions in Theorem 2.3, it holds that

$$
\int_{0}^{t} \int_{0}^{1} u^{2} d x d s \leq C
$$

where $C$ is a positive constant, independent of $t$.

Proof. Since $u(1, t)=0$, we have

$$
\begin{aligned}
|u(x, t)| & \leq \int_{x}^{1}\left|u_{y}(y, t)\right| d y \\
& =\int_{x}^{1}\left|u_{y}(y, t)\right| c^{\frac{\theta}{2}} Q^{\frac{1+\theta}{2}} c^{-\frac{\theta}{2}} Q^{-\frac{1+\theta}{2}} d y \\
& \leq\left(\int_{x}^{1} c^{\theta} Q^{1+\theta} u_{y}^{2} d y\right)^{\frac{1}{2}}\left(\int_{x}^{1} c^{-\theta} Q^{-(1+\theta)} d y\right)^{\frac{1}{2}} \\
& \leq C\left(\int_{x}^{1} c^{\theta} Q^{1+\theta} u_{y}^{2} d y\right)^{\frac{1}{2}}\left(\int_{x}^{1} c^{-(1+\theta)} Q^{-(1+\theta)} d y\right)^{\frac{1}{2}}
\end{aligned}
$$

It is easy to see from (2.6), (3.6) and $\left(A_{1}\right)$

$$
\int_{0}^{1} \int_{x}^{1}(c Q)^{-(1+\theta)} d y d x \leq C \int_{0}^{1} \int_{x}^{1} y^{-\frac{1+\theta}{\gamma}} d y d x \leq C
$$

provided $\theta<2 \gamma-1$, and

$$
\int_{0}^{1} u^{2} d x \leq \int_{0}^{1} c^{\theta} Q^{1+\theta} u_{x}^{2} d x \int_{0}^{1} \int_{x}^{1}(c Q)^{-(1+\theta)} d y d x \leq C \int_{0}^{1} c^{\theta} Q^{1+\theta} u_{x}^{2} d x
$$

Using (3.4), we get (3.12) immediately. This completes the proof of Lemma 3.4.

Next lemma plays a crucial role in dealing with the frictional force $-h(Q) u|u|$. The proof is based on recurrence method. 
Lemma 3.5. Under the conditions in Theorem 2.3, it holds that

$$
\int_{0}^{1}|u|^{5} d x+\int_{0}^{t} \int_{0}^{1} h(Q)|u|^{6} d x d s+\int_{0}^{t} \int_{0}^{1} c^{\theta} Q^{1+\theta}|u|^{3} u_{x}^{2} d x d s \leq C
$$

where $C$ is a positive constant, independent of $t$. Moveover,

$$
\int_{0}^{t} \int_{0}^{1}|u|^{3} d x d s \leq C, \quad \int_{0}^{t} \int_{0}^{1}|u|^{4} d x d s \leq C .
$$

Proof. Multiplying (2.2) $)_{3}$ by $p|u|^{p-2} u(p \geq 2)$ and integrating the result equation over $[0,1]$ with respect to $x$,

$$
\begin{aligned}
& \frac{d}{d t} \int_{0}^{1}|u|^{p} d x+p \int_{0}^{1} h(Q)|u|^{p-1} u^{2} d x+p(p-1) \int_{0}^{1} c^{\theta} Q^{1+\theta}|u|^{p-2} u_{x}^{2} d x \\
= & p(p-1) \int_{0}^{1}|u|^{p-2} u_{x}(c Q)^{\gamma} d x+g p \int_{0}^{1}|u|^{p-2} u d x \\
\leq & \frac{p(p-1)}{2} \int_{0}^{1} c^{\theta} Q^{1+\theta}|u|^{p-2} u_{x}^{2} d x+C \int_{0}^{1}|u|^{p-2} c^{2 \gamma-\theta} Q^{2 \gamma-\theta-1} d x+g p \int_{0}^{1}|u|^{p-1} d x \\
\leq & \frac{p(p-1)}{2} \int_{0}^{1} c^{\theta} Q^{1+\theta}|u|^{p-2} u_{x}^{2} d x+C \int_{0}^{1}|u|^{p-2} d x+g p \int_{0}^{1}|u|^{p-1} d x,
\end{aligned}
$$

where we have used the fact

$$
c^{2 \gamma-\theta} Q^{2 \gamma-\theta-1}=c(c Q)^{2 \gamma-\theta-1} \leq C x^{2-\frac{\theta+1}{\gamma}} \leq C
$$

Then integrating (3.16) over $[0, t]$ with respect to $t$, one has

$$
\begin{aligned}
& \int_{0}^{1}|u|^{p} d x+p \int_{0}^{t} \int_{0}^{1} h(Q)|u|^{p-1} u^{2} d x d s+\frac{p(p-1)}{2} \int_{0}^{t} \int_{0}^{1} c^{\theta} Q^{1+\theta}|u|^{p-2} u_{x}^{2} d x d s \\
& \leq \int_{0}^{1}\left|u_{0}\right|^{p} d x+C \int_{0}^{t} \int_{0}^{1}|u|^{p-2} d x d s+C \int_{0}^{t} \int_{0}^{1}|u|^{p-1} d x d s .
\end{aligned}
$$

Now taking $p=4$ in (3.17), we have

$$
\begin{aligned}
& \int_{0}^{1}|u|^{4} d x+4 \int_{0}^{t} \int_{0}^{1} h(Q)|u|^{3} u^{2} d x d s+6 \int_{0}^{t} \int_{0}^{1} c^{\theta} Q^{1+\theta}|u|^{2} u_{x}^{2} d x d s \\
\leq & C \int_{0}^{t} \int_{0}^{1}|u|^{3} d x d s+C,
\end{aligned}
$$

where we have used Lemma 3.4 and the assumption $\left(A_{2}\right)$.

Next we must estimate the first term of the right-hand side in (3.18) by using the same method as Lemma 3.4. Since $u(1, t)=0$, we have

$$
|u|^{\frac{3}{2}} \leq\left|\int_{x}^{1}\left(|u|^{\frac{3}{2}}\right) y d y\right| \leq C \int_{x}^{1}|u|^{\frac{1}{2}}\left|u_{y}\right| d y \leq C\left(\int_{0}^{1} c^{\theta} Q^{1+\theta} u_{y}^{2} d y\right)^{\frac{1}{2}}\left(\int_{x}^{1} c^{-\theta} Q^{-1-\theta}|u| d y\right)^{\frac{1}{2}}
$$


It is easy to see

$$
\begin{aligned}
\int_{0}^{1}|u|^{3} d x & \leq C\left(\int_{0}^{1} c^{\theta} Q^{1+\theta} u_{y}^{2} d y\right)\left(\int_{0}^{1} \int_{x}^{1} c^{-\theta} Q^{-1-\theta}|u| d y d x\right) \\
& \leq C\left(\int_{0}^{1} c^{\theta} Q^{1+\theta} u_{y}^{2} d y\right)\left(\int_{0}^{1} \int_{x}^{1} c(c Q)^{-1-\theta}|u| d y d x\right) \\
& \leq C\left(\int_{0}^{1} c^{\theta} Q^{1+\theta} u_{y}^{2} d y\right)\left(\int_{0}^{1} \int_{x}^{1} y^{-\frac{1+\theta}{\gamma}+\alpha}|u| d y d x\right) \\
& \leq C\left(\int_{0}^{1} c^{\theta} Q^{1+\theta} u_{y}^{2} d y\right)\left(\int_{0}^{1} \int_{x}^{1} y^{-\frac{4(1+\theta)}{3 \gamma}+\frac{4 \alpha}{3}} d y d x\right)^{\frac{3}{4}}\left(\int_{0}^{1} u^{4} d x\right)^{\frac{1}{4}} \\
& \leq C \int_{0}^{1} c^{\theta} Q^{1+\theta} u_{y}^{2} d y\left(\int_{0}^{1} u^{4} d x\right)^{\frac{1}{4}}
\end{aligned}
$$

where we have used $\theta<\left(\frac{3}{2}+\alpha\right) \gamma-1$.

Integrating (3.19) over $[0, t]$ and using (3.4), we get

$$
\begin{aligned}
\int_{0}^{t} \int_{0}^{1}|u|^{3} d x d s & \leq C \sup _{t \geq 0}\left(\int_{0}^{1} u^{4} d x\right)^{\frac{1}{4}} \int_{0}^{t} \int_{0}^{1} c^{\theta} Q^{1+\theta} u_{y}^{2} d y d s \\
& \leq C \epsilon_{0} \sup _{t \geq 0}\left(\int_{0}^{1} u^{4} d x\right)^{\frac{1}{4}} .
\end{aligned}
$$

Substituting (3.20) into (3.18), we have

$$
\begin{aligned}
& \int_{0}^{1}|u|^{4} d x+\int_{0}^{t} \int_{0}^{1} h(Q)|u|^{3} u^{2} d x d s+\int_{0}^{t} \int_{0}^{1} c^{\theta} Q^{1+\theta}|u|^{2} u_{x}^{2} d x d s \\
\leq & C+C \epsilon_{0} \sup _{t \geq 0}\left(\int_{0}^{1} u^{4} d x\right)^{\frac{1}{4}} .
\end{aligned}
$$

Young inequality and (3.21) imply that

$$
\int_{0}^{1}|u|^{4} d x \leq C
$$

Substituting (3.22) into (3.20), we have

$$
\int_{0}^{t} \int_{0}^{1}|u|^{3} d x d s \leq C .
$$

Combining (3.18) and (3.23), we have

$$
\int_{0}^{1}|u|^{4} d x+\int_{0}^{t} \int_{0}^{1} h(Q)|u|^{3} u^{2} d x d s+\int_{0}^{t} \int_{0}^{1} c^{\theta} Q^{1+\theta}|u|^{2} u_{x}^{2} d x d s \leq C .
$$

Taking $p=5$ in (3.17), we have

$$
\begin{aligned}
& \int_{0}^{1}|u|^{5} d x+5 \int_{0}^{t} \int_{0}^{1} h(Q)|u|^{4} u^{2} d x d s+10 \int_{0}^{t} \int_{0}^{1} c^{\theta} Q^{1+\theta}|u|^{3} u_{x}^{2} d x d s \\
\leq & \int_{0}^{1}\left|u_{0}\right|^{5} d x+C \int_{0}^{t} \int_{0}^{1}|u|^{3} d x d s+C \int_{0}^{t} \int_{0}^{1}|u|^{4} d x d s .
\end{aligned}
$$


In order to complete the proof of Lemma 3.5, it suffices to estimate the third term of the right-hand side in (3.25) by using the same method as Lemma 3.4. In fact

$$
|u|^{2} \leq\left|\int_{x}^{1}\left(|u|^{2}\right)_{y} d y\right| \leq C \int_{x}^{1}|u|\left|u_{y}\right| d y \leq C\left(\int_{0}^{1} c^{\theta} Q^{1+\theta} u_{y}^{2} d y\right)^{\frac{1}{2}}\left(\int_{x}^{1} c^{-\theta} Q^{-1-\theta} u^{2} d y\right)^{\frac{1}{2}},
$$

which implies

$$
\begin{aligned}
\int_{0}^{1}|u|^{4} d x & \leq C\left(\int_{0}^{1} c^{\theta} Q^{1+\theta} u_{y}^{2} d y\right)\left(\int_{0}^{1} \int_{x}^{1} c^{-\theta} Q^{-1-\theta} u^{2} d y d x\right) \\
& \leq C\left(\int_{0}^{1} c^{\theta} Q^{1+\theta} u_{y}^{2} d y\right)\left(\int_{0}^{1} \int_{x}^{1} y^{-\frac{(1+\theta)}{\gamma}+\alpha} u^{2} d y d x\right) \\
& \leq C\left(\int_{0}^{1} c^{\theta} Q^{1+\theta} u_{y}^{2} d y\right)\left(\int_{0}^{1} \int_{x}^{1} y^{-\frac{5(1+\theta)}{3 \gamma}+\frac{5 \alpha}{3}} d y d x\right)^{\frac{3}{5}}\left(\int_{0}^{1}|u|^{5} d x\right)^{\frac{2}{5}} \\
& \leq C\left(\int_{0}^{1} c^{\theta} Q^{1+\theta} u_{y}^{2} d y\right)\left(\int_{0}^{1}|u|^{5} d x\right)^{\frac{2}{5}} \cdot
\end{aligned}
$$

Here we have used $\theta<\left(\frac{6}{5}+\alpha\right) \gamma-1$.

Integrating (3.26) over $[0, t]$ and using (3.4), we get

$$
\begin{aligned}
\int_{0}^{t} \int_{0}^{1}|u|^{4} d x d s & \leq C \sup _{t \geq 0}\left(\int_{0}^{1}|u|^{5} d x\right)^{\frac{2}{5}} \int_{0}^{t} \int_{0}^{1} c^{\theta} Q^{1+\theta} u_{y}^{2} d y d s \\
& \leq C \epsilon_{0} \sup _{t \geq 0}\left(\int_{0}^{1}|u|^{5} d x\right)^{\frac{2}{5}} .
\end{aligned}
$$

Substituting (3.27) into (3.25) and using (3.23) and Young inequality, we have

$$
\int_{0}^{1}|u|^{5} d x \leq C
$$

Substituting (3.28) into (3.27), we have

$$
\int_{0}^{t} \int_{0}^{1}|u|^{4} d x d s \leq C .
$$

(3.25), (3.23) and (3.29) imply (3.14). This completes the proof of Lemma 3.5.

Lemma 3.6. Under the conditions in Theorem 2.3, it holds that

$$
\int_{0}^{1} u_{t}^{2} d x+\int_{0}^{t} \int_{0}^{1} h(Q)|u| u_{t}^{2} d x d s+\int_{0}^{t} \int_{0}^{1} c^{\theta} Q^{1+\theta} u_{x t}^{2} d x d s \leq C
$$

where $C$ is a positive constant, independent of $t$.

Proof. Differentiating (2.2) 3 with respect to $t$, multiplying $u_{t}$ and integrating over $[0,1]$ about $x$, we have

$$
\frac{1}{2} \frac{d}{d t} \int_{0}^{1} u_{t}^{2} d x+\int_{0}^{1}\left((c Q)^{\gamma}\right)_{x t} u_{t} d x=\int_{0}^{1}\left(c^{\theta} Q^{1+\theta} u_{x}\right)_{x t} u_{t} d x-\int_{0}^{1}(h(Q) u|u|)_{t} u_{t} d x
$$


Using the boundary conditions (2.4), and integrating by parts, we have

$$
\int_{0}^{1}\left((c Q)^{\gamma}\right)_{x t} u_{t} d x=-\int_{0}^{1}\left((c Q)^{\gamma}\right)_{t} u_{x t} d x=-\gamma \int_{0}^{1} c^{\gamma} Q^{\gamma-1} Q_{t} u_{x t} d x=\gamma \rho_{l} \int_{0}^{1} c^{\gamma} Q^{\gamma+1} u_{x} u_{x t} d x
$$

and

$$
\begin{aligned}
\int_{0}^{1}\left(c^{\theta} Q^{1+\theta} u_{x}\right)_{x t} u_{t} d x & =-\int_{0}^{1}\left(c^{\theta} Q^{1+\theta} u_{x}\right)_{t} u_{x t} d x \\
& =-\int_{0}^{1} c^{\theta}\left(Q^{1+\theta}\right)_{t} u_{x} u_{x t} d x-\int_{0}^{1} c^{\theta} Q^{1+\theta} u_{x t}^{2} d x \\
& =(1+\theta) \rho_{l} \int_{0}^{1} c^{\theta} Q^{2+\theta} u_{x}^{2} u_{x t} d x-\int_{0}^{1} c^{\theta} Q^{1+\theta} u_{x t}^{2} d x
\end{aligned}
$$

Substituting (3.32) and (3.33) into (3.31), and using Cauchy inequality, we get

$$
\begin{aligned}
& \frac{1}{2} \frac{d}{d t} \int_{0}^{1} u_{t}^{2} d x+\int_{0}^{1} c^{\theta} Q^{1+\theta} u_{x t}^{2} d x+2 \int_{0}^{1} h(Q)|u| u_{t}^{2} d x \\
= & (1+\theta) \rho_{l} \int_{0}^{1} c^{\theta} Q^{2+\theta} u_{x}^{2} u_{x t} d x-\gamma \rho_{l} \int_{0}^{1} c^{\gamma} Q^{\gamma+1} u_{x} u_{x t} d x+2 f \rho_{l}^{3} \int_{0}^{1} \frac{Q^{3}}{(1+Q)^{3}} u|u| u_{x} u_{t} d x \\
\leq & \frac{1}{2} \int_{0}^{1} c^{\theta} Q^{1+\theta} u_{x t}^{2} d x+C \int_{0}^{1} c^{\theta} Q^{\theta+3} u_{x}^{4} d x+C \int_{0}^{1} c^{2 \gamma-\theta} Q^{2 \gamma-\theta+1} u_{x}^{2} d x \\
& +\int_{0}^{1} h(Q)|u| u_{t}^{2} d x+C \int_{0}^{1} \frac{Q^{6}}{h(Q)(1+Q)^{6}}|u|^{3} u_{x}^{2} d x,
\end{aligned}
$$

which implies

$$
\begin{aligned}
& \frac{1}{2} \frac{d}{d t} \int_{0}^{1} u_{t}^{2} d x+\frac{1}{2} \int_{0}^{1} c^{\theta} Q^{1+\theta} u_{x t}^{2} d x+\int_{0}^{1} h(Q)|u| u_{t}^{2} d x \\
\leq & C \int_{0}^{1} c^{2 \gamma-\theta} Q^{2 \gamma-\theta+1} u_{x}^{2} d x+C \int_{0}^{1} c^{\theta} Q^{\theta+3} u_{x}^{4} d x+C \int_{0}^{1} \frac{Q^{4}}{(1+Q)^{4}}|u|^{3} u_{x}^{2} d x \\
\leq & C \int_{0}^{1}\left(c^{2 \gamma-2 \theta} Q^{2 \gamma-2 \theta}\right) c^{\theta} Q^{1+\theta} u_{x}^{2} d x+C \int_{0}^{1} c^{\theta} Q^{1+\theta} u_{x}^{2}\left(Q u_{x}\right)^{2} d x+C \int_{0}^{1} c^{-\theta} Q^{3-\theta} c^{\theta} Q^{1+\theta}|u|^{3} u_{x}^{2} d x \\
\leq & C \int_{0}^{1} c^{\theta} Q^{1+\theta} u_{x}^{2} d x+C \int_{0}^{1} c^{\theta} Q^{1+\theta} u_{x}^{2}\left(Q u_{x}\right)^{2} d x+C \int_{0}^{1} c^{\theta} Q^{1+\theta}|u|^{3} u_{x}^{2} d x
\end{aligned}
$$

where we have used the fact

$$
c^{-\theta} Q^{3-\theta} \leq C x^{\frac{3(1-\alpha \gamma)}{\gamma}-\frac{\theta}{\gamma}} \leq C
$$


which follows from $\theta \leq 1-\alpha \gamma$ and (2.6). By (2.6) and Lemma 3.5, we get

$$
\begin{aligned}
\left(Q u_{x}\right)^{2} & =\left(c^{\theta} Q^{1+\theta} u_{x}\right)^{2}(c Q)^{-2 \theta} \\
& =(c Q)^{-2 \theta}\left(\int_{0}^{x} u_{t} d y+(c Q)^{\gamma}-\left(c Q_{\infty}\right)^{\gamma}+\int_{0}^{x} h(Q) u|u| d y\right)^{2} \\
& \leq C(c Q)^{-2 \theta}\left\{\left(\int_{0}^{x} u_{t} d y\right)^{2}+(c Q)^{2 \gamma}+\left(c Q_{\infty}\right)^{2 \gamma}+\left(\int_{0}^{x} h(Q) u|u| d y\right)^{2}\right\} \\
& \leq C(c Q)^{-2 \theta} x \int_{0}^{1} u_{t}^{2} d x+C(c Q)^{2 \gamma-2 \theta}+C(c Q)^{-2 \theta} x^{2}+C(c Q)^{-2 \theta} x \int_{0}^{1} u^{4} d x \\
& \leq C \int_{0}^{1} u_{t}^{2} d x+C .
\end{aligned}
$$

Here we have used the fact

$$
(c Q)^{-2 \theta} x \leq C x^{1-\frac{2 \theta}{\gamma}} \leq C,
$$

which follows from $\theta \leq \frac{\gamma}{2}$. Then it follows from (3.34), (3.35) and Lemma 3.5 that

$$
\begin{aligned}
& \frac{d}{d t} \int_{0}^{1} u_{t}^{2} d x+\int_{0}^{1} c^{\theta} Q^{1+\theta} u_{x t}^{2} d x+2 \int_{0}^{1} h(Q)|u| u_{t}^{2} d x \\
\leq & C \int_{0}^{1} c^{\theta} Q^{1+\theta} u_{x}^{2} d x \int_{0}^{1} u_{t}^{2} d x+C \int_{0}^{1} c^{\theta} Q^{1+\theta} u_{x}^{2} d x .
\end{aligned}
$$

According to assumption $\left(A_{3}\right)$, and taking care of $(2.2)_{3}$, we obtain $\int_{0}^{1} u_{t}^{2}(x, 0) d x \leq C$. Then Gronwall inequality and (3.4) imply that

$$
\int_{0}^{1} u_{t}^{2} d x \leq C+C \exp \left(C \int_{0}^{t} \int_{0}^{1} c^{\theta} Q^{1+\theta} u_{x}^{2} d x d s\right) \leq C,
$$

and (3.30) follows. This proves Lemma 3.6.

Corollary 3.7. It follows from (3.30) and (3.35) that

$$
\left\|Q u_{x}\right\|_{L^{\infty}([0,1] \times[0, \infty))} \leq C,
$$

where $C$ is a positive constant, independent of $t$.

Lemma 3.8. Assume the conditions in Theorem 2.3 hold, and let $\beta$ be a fixed constant. (i) if $\beta>0$, then

$$
\int_{0}^{t} \int_{0}^{1}\left((c Q)^{\gamma}-\left(c Q_{\infty}\right)^{\gamma}\right)\left((c Q)^{\beta}-\left(c Q_{\infty}\right)^{\beta}\right) d x d s \leq C
$$

(ii) if $\beta \in W$, then

$$
\int_{0}^{t} \int_{0}^{1}\left((c Q)^{\gamma}-\left(c Q_{\infty}\right)^{\gamma}\right)\left(\left(c Q_{\infty}\right)^{-\beta}-(c Q)^{-\beta}\right) d x d s \leq C
$$

where $C$ is a positive constant, independent of $t . W=(0,2(1-\gamma \alpha)] \cap\left(0, \frac{(1-\alpha) \gamma+1-\theta}{2}\right]$. 
Proof. We only prove (ii) here, since the proof of (i) is almost the same with the later.

Due to (3.2), we have

$$
\begin{aligned}
& \int_{0}^{t} \int_{0}^{1}\left((c Q)^{\gamma}-\left(c Q_{\infty}\right)^{\gamma}\right)\left(\left(c Q_{\infty}\right)^{-\beta}-(c Q)^{-\beta}\right) d x d s \\
= & -\frac{1}{\theta \rho_{l}} \int_{0}^{t} \int_{0}^{1}\left((c Q)^{\theta}\right)_{t}\left(\left(c Q_{\infty}\right)^{-\beta}-(c Q)^{-\beta}\right) d x d s \\
& -\int_{0}^{t} \int_{0}^{1}\left(\left(c Q_{\infty}\right)^{-\beta}-(c Q)^{-\beta}\right)\left(\int_{0}^{x} u d y\right)_{t} d x d s \\
& -\int_{0}^{t} \int_{0}^{1}\left(\int_{0}^{x} h(Q) u|u| d y\right)\left(\left(c Q_{\infty}\right)^{-\beta}-(c Q)^{-\beta}\right) d x d s \\
= & I_{1}+I_{2}+I_{3},
\end{aligned}
$$

where

$$
\begin{aligned}
I_{1} & =-\frac{1}{\rho_{l} \theta} \int_{0}^{t} \int_{0}^{1}\left((c Q)^{\theta}\right)_{t}\left(\left(c Q_{\infty}\right)^{-\beta}-(c Q)^{-\beta}\right) d x d s \\
& =\frac{1}{\rho_{l} \theta} \int_{0}^{1}\left(\left(c Q_{0}\right)^{\theta}-(c Q)^{\theta}\right)\left(c Q_{\infty}\right)^{-\beta} d x+\frac{1}{\rho_{l}} \int_{0}^{1} \int_{0}^{t}(c Q)^{\theta-\beta-1}(c Q)_{t} d s d x \\
& =I_{1}^{(1)}+I_{1}^{(2)} .
\end{aligned}
$$

By using $\left(A_{1}\right),(\underline{2.6})$ and (3.6), one easily gets

$$
\left|I_{1}^{(1)}\right| \leq C \int_{0}^{1} x^{\frac{\theta-\beta}{\gamma}} d x \leq C
$$

provided $\beta<\theta+\gamma$.

Next we estimate $I_{1}^{(2)}$ by two cases.

Case 1: $\beta \neq \theta$.

In this case, if $\beta<\theta+\gamma$, similarly to (3.39), we have

$$
\left|I_{1}^{(2)}\right|=\left|\frac{1}{\rho_{l}(\theta-\beta)} \int_{0}^{1}\left((c Q)^{\theta-\beta}-\left(c Q_{0}\right)^{\theta-\beta}\right) d x\right| \leq C .
$$

Case 2: $\beta=\theta$.

In this case, also by $(A)_{1},(3.6)$, we have

$$
\left|I_{1}^{(2)}\right|=\left|\frac{1}{\rho_{l}} \int_{0}^{1}\left(\ln (c Q)-\ln \left(c Q_{0}\right)\right) d x\right| \leq C \int_{0}^{1}|\ln x| d x \leq C .
$$

Hence, if $\beta<\theta+\gamma$, we have

$$
\left|I_{1}\right| \leq C
$$


Now we estimate $I_{2}$ as follows:

$$
\begin{aligned}
I_{2}= & -\int_{0}^{1}\left(\left(c Q_{\infty}\right)^{-\beta}-(c Q)^{-\beta}\right)\left(\int_{0}^{x} u d y\right) d x+\int_{0}^{1}\left(\left(c Q_{\infty}\right)^{-\beta}-\left(c Q_{0}\right)^{-\beta}\right)\left(\int_{0}^{x} u_{0} d y\right) d x \\
& +\int_{0}^{t} \int_{0}^{1}\left(\left(c Q_{\infty}\right)^{-\beta}-(c Q)^{-\beta}\right)_{t}\left(\int_{0}^{x} u d y\right) d x d s \\
= & I_{2}^{(1)}+I_{2}^{(2)},
\end{aligned}
$$

where

$$
\begin{aligned}
\left|I_{2}^{(1)}\right| & =\left|-\int_{0}^{1}\left(\left(c Q_{\infty}\right)^{-\beta}-(c Q)^{-\beta}\right)\left(\int_{0}^{x} u d y\right) d x+\int_{0}^{1}\left(\left(c Q_{\infty}\right)^{-\beta}-\left(c Q_{0}\right)^{-\beta}\right)\left(\int_{0}^{x} u_{0} d y\right) d x\right| \\
& \leq C \int_{0}^{1} x^{-\frac{\beta}{\gamma}} x^{\frac{1}{2}}\left\{\left(\int_{0}^{1} u^{2} d x\right)^{\frac{1}{2}}+\left(\int_{0}^{1} u_{0}^{2} d x\right)^{\frac{1}{2}}\right\} d x \\
& \leq C \int_{0}^{1} x^{\frac{1}{2}-\frac{\beta}{\gamma}} d x \leq C .
\end{aligned}
$$

Here, $\beta<\frac{3}{2} \gamma,\left(A_{1}\right)$, (2.6), (3.4), (3.6) and Hölder inequality were used.

From (2.2) 2 , Hölder inequality, (3.4), (3.6) and (3.12), we have

$$
\begin{aligned}
\left|I_{2}^{(2)}\right| & =\int_{0}^{t} \int_{0}^{1}\left(\left(c Q_{\infty}\right)^{-\beta}-(c Q)^{-\beta}\right)_{t}\left(\int_{0}^{x} u d y\right) d x d s \\
& \leq C \int_{0}^{t} \int_{0}^{1} c^{-\beta} Q^{1-\beta}\left|u_{x}\right| x^{\frac{1}{2}}\left(\int_{0}^{x} u^{2} d y\right)^{\frac{1}{2}} d x d s \\
& \leq C\left(\int_{0}^{t} \int_{0}^{1} x c^{-\theta-2 \beta} Q^{1-\theta-2 \beta} c^{\theta} Q^{1+\theta} u_{x}^{2} d x d s\right)^{\frac{1}{2}}\left(\int_{0}^{t} \int_{0}^{1} u^{2} d x d s\right)^{\frac{1}{2}} \\
& \leq C \sup _{[0,1] \times[0, \infty)}\left(x^{1-\alpha}(c Q)^{1-\theta-2 \beta}\right)^{\frac{1}{2}}\left(\int_{0}^{t} \int_{0}^{1} c^{\theta} Q^{1+\theta} u_{x}^{2} d x d s\right)^{\frac{1}{2}} \\
& \leq C,
\end{aligned}
$$

provided $\beta \leq \frac{(1-\alpha) \gamma-\theta+1}{2}$.

Next, we estimate $I_{3}$,

$$
\begin{aligned}
I_{3} & =\int_{0}^{t} \int_{0}^{1}\left(\int_{0}^{x} h(Q) u|u| d y\right)\left(\left(c Q_{\infty}\right)^{-\beta}-(c Q)^{-\beta}\right) d x d s \\
& \leq \int_{0}^{t} \int_{0}^{1}\left(\int_{0}^{x} h(Q) u|u| d y\right)\left\{\left(c Q_{\infty}\right)^{-\beta}+(c Q)^{-\beta}\right\} d x d s \\
& =I_{3}^{(1)}+I_{3}^{(2)} .
\end{aligned}
$$

Because of the similarity of $I_{3}^{(1)}$ and $I_{3}^{(2)}$, we only estimate one of them,

$$
\left|I_{3}^{(1)}\right| \leq \sup \left[\left(c Q_{\infty}\right)^{-\beta} x^{\frac{2}{\gamma}-2 \alpha}\right] \int_{0}^{t} \int_{0}^{1} u^{2} d x d s \leq C
$$


provided $\beta \leq 2-2 \gamma \alpha$.

$\gamma>1$ and $\theta \in\left(0, \frac{\gamma}{2}\right] \cap(0, \gamma-1] \cap(0,1-\alpha \gamma]$ implies that $1-\alpha \gamma \in(0,2(1-\gamma \alpha)] \cap\left(0, \frac{(1-\alpha) \gamma+1-\theta}{2}\right] \cap$ $(0, \gamma+\theta) \cap\left(0, \frac{3}{2} \gamma\right)=(0,2(1-\gamma \alpha)] \cap\left(0, \frac{(1-\alpha) \gamma+1-\theta}{2}\right]$. Hence, $W$ is not empty, it follows from (3.39)-(3.44) that (3.38) holds. This completes the proof of Lemma 3.8.

In the next lemma, we give the uniform estimate of the derivative of the function $(c Q)(x, t)$ in a appropriate weighted $L^{2}$ space which is a little different from [8, 26]. Similar estimate for Navier-Stokes equations has been established by Zhang and Fang in $[8]$ for $\theta \in(0, \gamma-1) \cap\left(0, \frac{\gamma}{2}\right]$, by Zhu and $\mathrm{Zi}$ in [26] for $\theta \in(0, \gamma-1] \cap\left(0, \frac{\gamma}{2}\right]$.

Lemma 3.9. Assume the conditions in Theorem 2.3 hold, then

$$
\int_{0}^{1} x^{\frac{(2+\alpha) \gamma-\theta-1}{\gamma}}\left((c Q)^{\theta}-\left(c Q_{\infty}\right)^{\theta}\right)_{x}^{2} d x+\int_{0}^{t} \int_{0}^{1} x^{\frac{(3+\alpha) \gamma-2 \theta-1}{\gamma}}\left((c Q)^{\theta}-\left(c Q_{\infty}\right)^{\theta}\right)_{x}^{2} d x d t \leq C
$$

where $C$ is a positive constant, independent of $t$.

Proof. From (3.3) and (2.6), we get

$$
\begin{aligned}
& \left(\left((c Q)^{\theta}\right)_{x}-\left(\left(c Q_{\infty}\right)^{\theta}\right)_{x}+\theta \rho_{l} u\right)_{t}+\gamma \rho_{l}(c Q)^{\gamma-\theta}\left(\left((c Q)^{\theta}\right)_{x}-\left(\left(c Q_{\infty}\right)^{\theta}\right)_{x}+\theta \rho_{l} u\right) \\
= & \theta \rho_{l}^{2} \gamma(c Q)^{\gamma-\theta} u+\theta \rho_{l} g\left(1-\frac{(c Q)^{\gamma-\theta}}{\left(c Q_{\infty}\right)^{\gamma-\theta}}\right)-\theta \rho_{l} h(Q) u|u| .
\end{aligned}
$$

Multiplying (13.46) by $x^{\frac{(2+\alpha) \gamma-\theta-1}{\gamma}}\left(\left((c Q)^{\theta}\right)_{x}-\left(\left(c Q_{\infty}\right)^{\theta}\right)_{x}+\theta \rho_{l} u\right)$, and integrating the result equation on $[0,1] \times[0, t]$, we get

$$
\begin{aligned}
& \frac{1}{2} \int_{0}^{1} x^{\frac{(2+\alpha) \gamma-\theta-1}{\gamma}}\left(\left((c Q)^{\theta}\right)_{x}-\left(\left(c Q_{\infty}\right)^{\theta}\right)_{x}+\theta \rho_{l} u\right)^{2} d x \\
& +\gamma \rho_{l} \int_{0}^{t} \int_{0}^{1}(c Q)^{\gamma-\theta} x^{\frac{(2+\alpha) \gamma-\theta-1}{\gamma}}\left(\left((c Q)^{\theta}\right)_{x}-\left(\left(c Q_{\infty}\right)^{\theta}\right)_{x}+\theta \rho_{l} u\right)^{2} d x d s \\
= & \frac{1}{2} \int_{0}^{1} x^{\frac{(2+\alpha) \gamma-\theta-1}{\gamma}}\left(\left(\left(c Q_{0}\right)^{\theta}\right)_{x}-\left(\left(c Q_{\infty}\right)^{\theta}\right)_{x}+\theta \rho_{l} u_{0}\right)^{2} d x \\
& +\int_{0}^{t} \int_{0}^{1} x^{\frac{(2+\alpha) \gamma-\theta-1}{\gamma}}\left(\left((c Q)^{\theta}\right)_{x}-\left(\left(c Q_{\infty}\right)^{\theta}\right)_{x}+\theta \rho_{l} u\right)\left(\theta \rho_{l}^{2} \gamma(c Q)^{\gamma-\theta} u+\theta \rho_{l} g\left(1-\frac{(c Q)^{\gamma-\theta}}{\left(c Q_{\infty}\right)^{\gamma-\theta}}\right)\right) d x d s \\
& +\int_{0}^{t} \int_{0}^{1} x^{\frac{(2+\alpha) \gamma-\theta-1}{\gamma}}\left(\left((c Q)^{\theta}\right)_{x}-\left(\left(c Q_{\infty}\right)^{\theta}\right)_{x}+\theta \rho_{l} u\right)\left(-\theta \rho_{l} h(Q) u|u|\right) d x d s
\end{aligned}
$$




$$
\begin{aligned}
\leq & C+\gamma \rho_{l} \frac{1}{2} \int_{0}^{t} \int_{0}^{1}(c Q)^{\gamma-\theta} x^{\frac{(2+\alpha) \gamma-\theta-1}{\gamma}}\left(\left((c Q)^{\theta}\right)_{x}-\left(\left(c Q_{\infty}\right)^{\theta}\right)_{x}+\theta \rho_{l} u\right)^{2} d x d s \\
& +C \int_{0}^{t} \int_{0}^{1}(c Q)^{\gamma-\theta} x^{\frac{(2+\alpha) \gamma-\theta-1}{\gamma}} u^{2} d x d s+C \int_{0}^{t} \int_{0}^{1} x^{\frac{(2+\alpha) \gamma-\theta-1}{\gamma}}(c Q)^{\theta-\gamma}\left(1-\frac{(c Q)^{\gamma-\theta}}{\left(c Q_{\infty}\right)^{\gamma-\theta}}\right)^{2} d x d s \\
& +C \int_{0}^{t} \int_{0}^{1} x^{\frac{(2+\alpha) \gamma-\theta-1}{\gamma}}(c Q)^{\theta-\gamma} h^{2}(Q) u^{4} d x d s \\
\leq & C+\frac{1}{2} \int_{0}^{t} \int_{0}^{1} \gamma \rho_{l}(c Q)^{\gamma-\theta} x^{\frac{(2+\alpha) \gamma-\theta-1}{\gamma}}\left(\left((c Q)^{\theta}\right)_{x}-\left(\left(c Q_{\infty}\right)^{\theta}\right)_{x}+\theta \rho_{l} u\right)^{2} d x d s \\
& +C \int_{0}^{t} \int_{0}^{1} x^{\frac{(3+\alpha) \gamma-2 \theta-1}{\gamma}} u^{2} d x d s+C \int_{0}^{t} \int_{0}^{1} x^{-\frac{(1-\alpha) \gamma+1}{\gamma}}\left((c Q)^{\gamma}-\left(c Q_{\infty}\right)^{\gamma}\right)^{2} d x d s \\
& +C \int_{0}^{t} \int_{0}^{1} x^{\frac{(1+\alpha) \gamma-1}{\gamma}} u^{4} d x d s,
\end{aligned}
$$

which implies

$$
\begin{aligned}
& \int_{0}^{1} x^{\frac{(2+\alpha) \gamma-\theta-1}{\gamma}}\left(\left((c Q)^{\theta}\right)_{x}-\left(\left(c Q_{\infty}\right)^{\theta}\right)_{x}+\theta \rho_{l} u\right)^{2} d x \\
& +\gamma \rho_{l} \int_{0}^{t} \int_{0}^{1}(c Q)^{\gamma-\theta} x^{\frac{(2+\alpha) \gamma-\theta-1}{\gamma}}\left(\left((c Q)^{\theta}\right)_{x}-\left(\left(c Q_{\infty}\right)^{\theta}\right)_{x}+\theta \rho_{l} u\right)^{2} d x d s \\
& \leq C+C \int_{0}^{t} \int_{0}^{1} x^{\frac{(3+\alpha) \gamma-2 \theta-1}{\gamma}} u^{2} d x d s+C \int_{0}^{t} \int_{0}^{1} x^{\frac{(1+\alpha) \gamma-1}{\gamma}} u^{4} d x d s \\
& +\int_{0}^{t} \int_{0}^{1} A(x, t)\left\{(c Q)^{\gamma}-\left(c Q_{\infty}\right)^{\gamma}\right\}\left\{(c Q)^{-\beta}-\left(c Q_{\infty}\right)^{-\beta}\right\} d x d s,
\end{aligned}
$$

where

$$
\begin{aligned}
A(x, t) & =\frac{x^{\frac{(2+\alpha) \gamma-\theta-1}{\gamma}}(c Q)^{\theta-\gamma}\left(c Q_{\infty}\right)^{2 \theta-2 \gamma}\left\{\left(c Q_{\infty}\right)^{\gamma-\theta}-(c Q)^{\gamma-\theta}\right\}^{2}}{\left\{(c Q)^{\gamma}-\left(c Q_{\infty}\right)^{\gamma}\right\}\left\{\left(c Q_{\infty}\right)^{-\beta}-(c Q)^{-\beta}\right\}} \\
& =\frac{x^{\frac{(2+\alpha) \gamma-\theta-1}{\gamma}}(c Q)^{\beta+\theta-\gamma}\left(c Q_{\infty}\right)^{\beta+2 \theta-2 \gamma}\left\{\left(c Q_{\infty}\right)^{\gamma-\theta}-(c Q)^{\gamma-\theta}\right\}^{2}}{\left\{(c Q)^{\gamma}-\left(c Q_{\infty}\right)^{\gamma}\right\}\left\{(c Q)^{\beta}-\left(c Q_{\infty}\right)^{\beta}\right\}} .
\end{aligned}
$$

It is easy to see that

$$
\begin{aligned}
|A(x, t)| & \leq C x^{\frac{(2+\alpha) \gamma-\theta-1}{\gamma}}\left(c Q_{\infty}\right)^{\beta-2 \gamma+\theta} \\
& \leq C x \frac{(2+\alpha) \gamma-\theta-1+\beta-2 \gamma+\theta}{\gamma} \\
& \leq C
\end{aligned}
$$

provided $\beta=1-\alpha \gamma$. Here we have used Lemma 3.4 and (3.15). Taking $\beta=1-\alpha \gamma$ in (3.38), and using Lemma 3.8, we obtain (3.45) immediately from (3.48).

This completes the proof of Lemma 3.9. 


\section{Asymptotic behavior}

In this section, based on the a priori estimates obtained in Section 3, we will consider the asymptotic behavior of the solution $((c Q)(x, t), u(x, t))$ to the initial boundary problem (2.2)(2.4). We will show both $(c Q)(x, t)$ and $u(x, t)$ converge to the stationary state uniformly as $t \rightarrow \infty$.

To apply the uniform estimates obtained above to study the asymptotic behavior of $(c Q)(x, t)$ and $u(x, t)$, we introduce the following lemma (cf. [17, 8,2$]$ ), and omit the details of the proof.

Lemma 4.1. Suppose that $y \in W_{l o c}^{1,1}\left(\mathbb{R}^{+}\right)$satisfies

$$
y=y_{1}^{\prime}+y_{2},
$$

and

$$
\left|y_{2}\right| \leq \sum_{i=1}^{n} \alpha_{i}, \quad\left|y^{\prime}\right| \leq \sum_{i=1}^{n} \beta_{i}, \quad \text { on } \mathbb{R}^{+},
$$

where $y_{1} \in W_{l o c}^{1,1}\left(\mathbb{R}^{+}\right), \lim _{s \rightarrow+\infty} y_{1}(s)=0$ and $\alpha_{i}, \beta_{i} \in L^{p_{i}}\left(\mathbb{R}^{+}\right)$for some $p_{i} \in[1, \infty), i=1, \cdots, n$. Then

$$
\lim _{s \rightarrow+\infty} y(s)=0
$$

Proof of Theorem 2.3.

At first, we consider the convergence of $u(x, t)$. To this end, motivated by [16], we introduce a function of $t$ as follows:

$$
f(t)=\int_{0}^{1} c^{\theta} Q_{\infty}^{1+\theta} u_{x}^{2} d x
$$

Then by Lemma 3.2 and Lemma 3.3, we get

$$
\int_{0}^{\infty}|f(t)| d t=\int_{0}^{\infty} \int_{0}^{1} c^{\theta} Q_{\infty}^{1+\theta} u_{x}^{2} d x d t \leq C .
$$

Furthermore, by Lemmas 3.2, 3.3, 3.6 and Cauchy inequality, we have

$$
\int_{0}^{\infty}\left|\frac{d f(t)}{d t}\right| d t \leq \int_{0}^{\infty} \int_{0}^{1} c^{\theta} Q_{\infty}^{1+\theta} u_{x}^{2} d x d t+\int_{0}^{\infty} \int_{0}^{1} c^{\theta} Q_{\infty}^{1+\theta} u_{x t}^{2} d x d t \leq C .
$$

It follows from Lemma 4.1 that

$$
\lim _{t \rightarrow \infty} \int_{0}^{1} c^{\theta} Q_{\infty}^{1+\theta} u_{x}^{2} d x=0
$$

Therefore, by Hölder inequality, we have for $x \in[\delta, 1]$ with $0<\delta<1$,

$$
\begin{aligned}
|u(x, t)| & =\left|\int_{x}^{1} u_{z}(z, t) d z\right|=\left|\int_{x}^{1} c^{\frac{\theta}{2}} Q_{\infty}^{\frac{1+\theta}{2}} u_{z}(z, t) c^{-\frac{\theta}{2}} Q_{\infty}^{-\frac{1+\theta}{2}} d z\right| \\
& \leq\left(\int_{0}^{1} c^{\theta} Q_{\infty}^{1+\theta} u_{x}^{2}(x, t) d x\right)^{\frac{1}{2}}\left(\int_{\delta}^{1} c^{-\theta} Q_{\infty}^{-(1+\theta)} d x\right)^{\frac{1}{2}} \\
& \leq C\left(\int_{0}^{1} c^{\theta} Q_{\infty}^{1+\theta} u_{x}^{2}(x, t) d x\right)^{\frac{1}{2}} \rightarrow 0
\end{aligned}
$$


as $t \rightarrow \infty$, i.e.,

$$
\lim _{t \rightarrow \infty} \sup _{x \in[\delta, 1]} u(x, t)=0 .
$$

Furthermore, when $\delta=0$,

$$
\int_{0}^{1} c^{-\theta} Q_{\infty}^{-(1+\theta)} d x \leq \int_{0}^{1} x^{-\frac{1+\theta}{\gamma}+\alpha} d x \leq C
$$

provided $\theta<\gamma-1$. Thus, when $\theta<\gamma-1$, we have

$$
\lim _{t \rightarrow \infty} \sup _{x \in[0,1]} u(x, t)=0 .
$$

Next we consider the convergence of $(c Q)(x, t)$. Firstly, we shall show that $(c Q)(x, t)$ tends to the stationary state $\left(c Q_{\infty}\right)(x)$ in the sense of integral as $t \rightarrow \infty$. The similar conclusion for Navier-Stokes equation was obtained in [8] and [2] before.

Taking $\beta=\gamma$ in (3.37), we have

$$
\int_{0}^{1}\left((c Q)^{\gamma}-\left(c Q_{\infty}\right)^{\gamma}\right)^{2} d x \in L^{1}\left(\mathbb{R}^{+}\right) .
$$

On the other hand, it is easy to see that

$$
\begin{aligned}
\left|\frac{d}{d t} \int_{0}^{1}\left((c Q)^{\gamma}-\left(c Q_{\infty}\right)^{\gamma}\right)^{2} d x\right| & =\left|\int_{0}^{1} 2\left((c Q)^{\gamma}-\left(c Q_{\infty}\right)^{\gamma}\right) \gamma c^{\gamma} Q^{\gamma-1} Q_{t} d x\right| \\
& =2 \gamma \rho_{l}\left|\int_{0}^{1}\left((c Q)^{\gamma}-\left(c Q_{\infty}\right)^{\gamma}\right) c^{\gamma} Q^{\gamma+1} u_{x} d x\right| \\
& \leq C\left(\int_{0}^{1} c^{2 \gamma-\theta} Q^{2 \gamma+1-\theta} d x\right)^{1 / 2}\left(\int_{0}^{1} c^{\theta} Q^{1+\theta} u_{x}^{2} d x\right)^{1 / 2} \\
& \leq C\left(\int_{0}^{1} c^{2 \gamma-\theta} Q^{2 \gamma-\theta} d x\right)^{1 / 2}\left(\int_{0}^{1} c^{\theta} Q^{1+\theta} u_{x}^{2} d x\right)^{1 / 2} \\
& \leq C\left(\int_{0}^{1} c^{\theta} Q^{1+\theta} u_{x}^{2} d x\right)^{1 / 2} .
\end{aligned}
$$

Then (4.4), (4.5) and Lemma 4.1 yield

$$
\lim _{t \rightarrow \infty} \int_{0}^{1}\left((c Q)^{\gamma}-\left(c Q_{\infty}\right)^{\gamma}\right)^{2} d x=0
$$

Consequently,

$$
\begin{aligned}
\int_{0}^{1}\left(c Q-c Q_{\infty}\right)^{2 \gamma} d x & =\int_{0}^{1} \frac{\left(c Q-c Q_{\infty}\right)^{2 \gamma}}{\left((c Q)^{\gamma}-\left(c Q_{\infty}\right)^{\gamma}\right)^{2}}\left((c Q)^{\gamma}-\left(c Q_{\infty}\right)^{\gamma}\right)^{2} d x \\
& \leq C \int_{0}^{1}\left((c Q)^{\gamma}-\left(c Q_{\infty}\right)^{\gamma}\right)^{2} d x \rightarrow 0, \text { as } t \rightarrow \infty
\end{aligned}
$$

For $q \in(0,2 \gamma)$, we have from (4.7) and Hölder inequality that

$$
\int_{0}^{1}\left|c Q-c Q_{\infty}\right|^{q} d x \leq C\left(\int_{0}^{1}\left(c Q-c Q_{\infty}\right)^{2 \gamma} d x\right)^{\frac{q}{2 \gamma}} \rightarrow 0
$$


On the other hand, for $q \in(2 \gamma, \infty)$, we have from (2.6) and (3.6) that

$$
\begin{aligned}
\int_{0}^{1}\left|c Q-c Q_{\infty}\right|^{q} d x & =\int_{0}^{1}\left|c Q-c Q_{\infty}\right|^{q-2 \gamma}\left(c Q-c Q_{\infty}\right)^{2 \gamma} d x \\
& \leq C \int_{0}^{1}\left(c Q-c Q_{\infty}\right)^{2 \gamma} d x \rightarrow 0
\end{aligned}
$$

Hence, by (4.8) and (4.9), we get

$$
\left\|\left(c Q-c Q_{\infty}\right)(\cdot, t)\right\|_{L^{q}} \rightarrow 0, \text { as } t \rightarrow \infty, \quad q \in(0, \infty)
$$

We are now in a position to show the uniform convergence of $(c Q)(x, t)$. To this end, choosing a positive number $k$ large enough, which is to be determined later, applying Hölder inequality, (2.6) and (3.6), we have by $(c Q)(0, t)=\left(c Q_{\infty}\right)(0)=0$

$$
\begin{aligned}
0 & \leq\left|(c Q)^{\theta}(x, t)-\left(c Q_{\infty}\right)^{\theta}(x)\right|^{k} \\
\leq & k \int_{0}^{x}\left|(c Q)^{\theta}-\left(c Q_{\infty}\right)^{\theta}\right|^{k-1}\left|\left((c Q)^{\theta}-\left(c Q_{\infty}\right)^{\theta}\right)_{x}\right| d y \\
\leq & k\left(\int_{0}^{1}\left(c Q_{\infty}\right)^{-\eta}\left|(c Q)^{\theta}-\left(c Q_{\infty}\right)^{\theta}\right|^{2 k-2} d x\right)^{\frac{1}{2}}\left(\int_{0}^{1}\left(c Q_{\infty}\right)^{\eta}\left((c Q)^{\theta}-\left(c Q_{\infty}\right)^{\theta}\right)_{x}^{2} d x\right)^{\frac{1}{2}} \\
= & k\left(\int_{0}^{1}\left(c Q_{\infty}\right)^{-\eta}\left|(c Q)^{\theta}-\left(c Q_{\infty}\right)^{\theta}\right|^{\frac{\eta}{\theta}}\left|(c Q)^{\theta}-\left(c Q_{\infty}\right)^{\theta}\right|^{2 k-2-\frac{\eta}{\theta}} d x\right)^{\frac{1}{2}} \\
& \quad\left(\int_{0}^{1}\left(c Q_{\infty}\right)^{\eta}\left((c Q)^{\theta}-\left(c Q_{\infty}\right)^{\theta}\right)_{x}^{2} d x\right)^{\frac{1}{2}} \\
\leq & C\left(\int_{0}^{1}\left|(c Q)^{\theta}-\left(c Q_{\infty}\right)^{\theta}\right|^{2 k-2-\frac{\eta}{\theta}} d x\right)^{\frac{1}{2}}\left(\int_{0}^{1}\left(c Q_{\infty}\right)^{\eta}\left((c Q)^{\theta}-\left(c Q_{\infty}\right)^{\theta}\right)_{x}^{2} d x\right)^{\frac{1}{2}},
\end{aligned}
$$

where $\eta=(2+\alpha) \gamma-\theta-1$. Note that

$$
\left|(c Q)^{\theta}-\left(c Q_{\infty}\right)^{\theta}\right| \leq C\left|c Q-c Q_{\infty}\right|^{\min \{\theta, 1\}}=C\left|c Q-c Q_{\infty}\right|^{\theta} .
$$

Now letting $2 k-2-\frac{\eta}{\theta}>0$, we deduce from (4.10) and (4.11) that

$$
\lim _{t \rightarrow \infty}\left|(c Q)^{\theta}(x, t)-\left(c Q_{\infty}\right)^{\theta}(x)\right|^{k}=0
$$

uniformly in $x \in[0,1]$, and (2.10) follows. This completes the proof of Theorem 2.3.

\section{$5 \quad$ Stabilization rate estimates}

In this section, using the method in [8], we will give the stabilization rate estimates of the weak solution $((c Q)(x, t), u(x, t))$ under the condition $\theta \in(0, \gamma-1) \cap\left(0, \frac{\gamma}{2}\right] \cap(0,1-\alpha \gamma]$. Compared with the corresponding results in [8, 26], the only difference is that we must deal with the frictional force $-h(Q) u|u|$. 
Lemma 5.1. Assume the conditions in Theorem 2.3 hold, we have

$$
\int_{0}^{1}\left(u^{2}+x^{1-\frac{3}{\gamma}}\left(c Q-c Q_{\infty}\right)^{2}\right) d x \leq \frac{C}{1+t}, \quad \forall t \geq 0
$$

and

$$
\int_{0}^{\infty} \int_{0}^{1}(1+t) c^{\theta} Q^{1+\theta} u_{x}^{2} d x d t \leq C .
$$

Proof. Multiplying $(2.2)_{3}$ by $(1+t) u$, integrating the result equation over $[0,1] \times[0, t]$, integrating by parts, we get

$$
\begin{aligned}
& (1+t) \int_{0}^{1}\left(\frac{1}{2} u^{2}+\int_{Q_{\infty}}^{Q} \frac{c^{\gamma}\left(h^{\gamma}-Q_{\infty}^{\gamma}\right)}{\rho_{l} h^{2}} d h\right) d x+\int_{0}^{t} \int_{0}^{1}(1+s) c^{\theta} Q^{1+\theta} u_{x}^{2} d x d s \\
& +\int_{0}^{t} \int_{0}^{1}(1+s) h(Q) u^{2}|u| d x d s \\
= & \int_{0}^{1}\left(\frac{1}{2} u_{0}^{2}+\int_{Q_{\infty}}^{Q_{0}} \frac{c^{\gamma}\left(h^{\gamma}-Q_{\infty}^{\gamma}\right)}{\rho_{l} h^{2}} d h\right) d x+\int_{0}^{t} \int_{0}^{1}\left(\frac{1}{2} u^{2}+\int_{Q_{\infty}}^{Q} \frac{c^{\gamma}\left(h^{\gamma}-Q_{\infty}^{\gamma}\right)}{\rho_{l} h^{2}} d h\right) d x d s \\
\leq & C+C \int_{0}^{t} \int_{0}^{1} x^{\frac{\beta-1}{\gamma}+\alpha}\left((c Q)^{\gamma}-\left(c Q_{\infty}\right)^{\gamma}\right)\left(\left(c Q_{\infty}\right)^{-\beta}-(c Q)^{-\beta}\right) d x d s,
\end{aligned}
$$

where we have used (3.4), Lemma 3.4 and the following fact

$$
\begin{aligned}
& \int_{0}^{t} \int_{0}^{1}\left(\int_{Q_{\infty}}^{Q} \frac{c^{\gamma}\left(h^{\gamma}-Q_{\infty}^{\gamma}\right)}{\rho_{l} h^{2}} d h\right) d x d s \\
\leq & C \int_{0}^{t} \int_{0}^{1} Q_{\infty}^{-2}\left|Q-Q_{\infty}\right|\left|(c Q)^{\gamma}-\left(c Q_{\infty}\right)^{\gamma}\right| d x d s \\
\leq & C \int_{0}^{t} \int_{0}^{1} c\left(c Q_{\infty}\right)^{-2}\left|c Q-c Q_{\infty}\right|\left|(c Q)^{\gamma}-\left(c Q_{\infty}\right)^{\gamma}\right| d x d s \\
\leq & C \int_{0}^{t} \int_{0}^{1} x^{\frac{\beta-1}{\gamma}+\alpha}\left((c Q)^{\gamma}-\left(c Q_{\infty}\right)^{\gamma}\right)\left(\left(c Q_{\infty}\right)^{-\beta}-(c Q)^{-\beta}\right) d x d s .
\end{aligned}
$$

Choose $\beta=1-\alpha \gamma$, then (5.1) and (5.2) are immediately obtained from Lemma 3.8. This completes the proof of Lemma 5.1.

Corollary 5.2. Assume the conditions in Theorem 2.3 hold, we have

$$
\int_{0}^{t} \int_{0}^{1}(1+s)|u|^{i} d x d s \leq C, \quad i=2,3,4 .
$$

Proof. The result can be easily obtained by (3.13), (3.19), (3.26) and Lemma 5.1 and the details are omitted.

Corollary 5.3. Assume the conditions in Theorem 2.3 hold, we have

$$
\int_{0}^{t} \int_{0}^{1}(1+s) x^{-\frac{\theta+1}{\gamma}+\alpha}\left((c Q)^{\gamma}-\left(c Q_{\infty}\right)^{\gamma}\right)^{2} d x d s \leq C
$$


Proof. Due to $(\underline{2.2})_{3}$, we have

$$
\begin{aligned}
& \int_{0}^{t} \int_{0}^{1}(1+s) x^{-\frac{\theta+1}{\gamma}+\alpha}\left((c Q)^{\gamma}-\left(c Q_{\infty}\right)^{\gamma}\right)^{2} d x d s \\
= & \int_{0}^{t} \int_{0}^{1}(1+s) x^{-\frac{\theta+1}{\gamma}+\alpha}\left((c Q)^{\gamma}-\left(c Q_{\infty}\right)^{\gamma}\right)\left(-\int_{0}^{x} u_{t} d y\right) d x d s \\
& +\int_{0}^{t} \int_{0}^{1}(1+s) x^{-\frac{\theta+1}{\gamma}+\alpha}\left((c Q)^{\gamma}-\left(c Q_{\infty}\right)^{\gamma}\right)\left(-\int_{0}^{x} h(Q) u|u| d y\right) d x d s \\
& +\int_{0}^{t} \int_{0}^{1}(1+s) x^{-\frac{\theta+1}{\gamma}+\alpha}\left((c Q)^{\gamma}-\left(c Q_{\infty}\right)^{\gamma}\right)\left(c^{\theta} Q^{1+\theta} u_{x}\right) d x d s \\
= & J_{1}+J_{2}+J_{3} .
\end{aligned}
$$

Next, we estimate terms on the right hand side of the above equality as follows:

$$
\begin{aligned}
& J_{1}=\int_{0}^{1} x^{-\frac{\theta+1}{\gamma}+\alpha}\left(\left(c Q_{0}\right)^{\gamma}-\left(c Q_{\infty}\right)^{\gamma}\right)\left(\int_{0}^{x} u_{0} d y\right) d x \\
& -(1+t) \int_{0}^{1} x^{-\frac{\theta+1}{\gamma}+\alpha}\left((c Q)^{\gamma}-\left(c Q_{\infty}\right)^{\gamma}\right)\left(\int_{0}^{x} u d y\right) d x \\
& +\int_{0}^{t} \int_{0}^{1} x^{-\frac{\theta+1}{\gamma}+\alpha}\left((c Q)^{\gamma}-\left(c Q_{\infty}\right)^{\gamma}\right)\left(\int_{0}^{x} u d y\right) d x d s \\
& -\int_{0}^{t} \int_{0}^{1}(1+s) x^{-\frac{\theta+1}{\gamma}+\alpha} \gamma \rho_{l} c^{\gamma} Q^{\gamma+1} u_{x}\left(\int_{0}^{x} u d y\right) d x d s \\
& \leq \int_{0}^{1} x^{\frac{1}{2}-\frac{\theta+1}{\gamma}+1+\alpha}\left(\int_{0}^{1} u_{0}^{2} d y\right)^{\frac{1}{2}} d x+(1+t) \int_{0}^{1} x^{\frac{1}{2}-\frac{\theta+1}{\gamma}+1-\frac{2}{\gamma}+\alpha}\left(c Q-c Q_{\infty}\right)^{2}\left(\int_{0}^{1} u^{2} d y\right)^{\frac{1}{2}} d x \\
& +C \int_{0}^{t} \int_{0}^{1}\left(\int_{0}^{x} u^{2} d y\right) d x d s+\int_{0}^{t} \int_{0}^{1} x^{1-2 \frac{1+\theta}{\gamma}+2 \alpha}\left((c Q)^{\gamma}-\left(c Q_{\infty}\right)^{\gamma}\right)^{2} d x d s \\
& +C \int_{0}^{t} \int_{0}^{1}(1+s) c^{\theta} Q^{1+\theta} u_{x}^{2} d x d s+C \int_{0}^{t} \int_{0}^{1}(1+s) x^{1-2 \frac{\theta+1}{\gamma}+2 \alpha} c^{2 \gamma-\theta} Q^{2 \gamma-\theta+1}\left(\int_{0}^{1} u^{2} d y\right) d x d s \\
& \leq C+C(1+t) \int_{0}^{1} x^{1-\frac{3}{\gamma}}\left(c Q-c Q_{\infty}\right)^{2} d x+C \int_{0}^{t} \int_{0}^{1} u^{2} d x d s \\
& +\int_{0}^{t} \int_{0}^{1} x^{2-2 \frac{1+\theta}{\gamma}+2 \alpha-\frac{\beta}{\gamma}}\left((c Q)^{\gamma}-\left(c Q_{\infty}\right)^{\gamma}\right)\left((c Q)^{\beta}-\left(c Q_{\infty}\right)^{\beta}\right) d x d s \\
& +C \int_{0}^{t} \int_{0}^{1}(1+s) x^{3-\frac{3 \theta+1}{\gamma}+\alpha}\left(\int_{0}^{1} u^{2} d y\right) d x d s \\
& \leq C,
\end{aligned}
$$

where we have used (3.4), Lemma 3.4, Lemma 5.1, Corollary 5.2 and $\beta=2(\gamma-1-\theta+\alpha \gamma)>0$ 
in Lemma 3.8.

$$
\begin{aligned}
J_{2} \leq & \frac{1}{4} \int_{0}^{t} \int_{0}^{1}(1+s) x^{-\frac{\theta+1}{\gamma}+\alpha}\left((c Q)^{\gamma}-\left(c Q_{\infty}\right)^{\gamma}\right)^{2} d x d s \\
& +C \int_{0}^{t} \int_{0}^{1}(1+s) x^{-\frac{\theta+1}{\gamma}+\alpha}\left(\int_{0}^{x} h(Q) u|u| d y\right)^{2} d x d s \\
\leq & \frac{1}{4} \int_{0}^{t} \int_{0}^{1}(1+s) x^{-\frac{\theta+1}{\gamma}+\alpha}\left((c Q)^{\gamma}-\left(c Q_{\infty}\right)^{\gamma}\right)^{2} d x d s+C \int_{0}^{t} \int_{0}^{1} x^{1-\frac{\theta+1}{\gamma}+\alpha}(1+s)\left(\int_{0}^{x} u^{4} d y\right) d x d s \\
\leq & \frac{1}{4} \int_{0}^{t} \int_{0}^{1}(1+s) x^{-\frac{\theta+1}{\gamma}+\alpha}\left((c Q)^{\gamma}-\left(c Q_{\infty}\right)^{\gamma}\right)^{2} d x d s+C \int_{0}^{t} \int_{0}^{1}(1+s) u^{4} d x d s \\
\leq & \frac{1}{4} \int_{0}^{t} \int_{0}^{1}(1+s) x^{-\frac{\theta+1}{\gamma}+\alpha}\left((c Q)^{\gamma}-\left(c Q_{\infty}\right)^{\gamma}\right)^{2} d x d s+C,
\end{aligned}
$$

where we have used Young inequality and the fact $\theta<\gamma-1$ in Corollary 5.2.

By using Young inequality and Lemma 5.1, we have

$$
\begin{aligned}
J_{3} \leq & \frac{1}{4} \int_{0}^{t} \int_{0}^{1}(1+s) x^{-\frac{\theta+1}{\gamma}+\alpha}\left((c Q)^{\gamma}-\left(c Q_{\infty}\right)^{\gamma}\right)^{2} d x d s \\
& +C \int_{0}^{t} \int_{0}^{1}(1+s) x^{-\frac{\theta+1}{\gamma}+\alpha}\left(c^{\theta} Q^{1+\theta}\right) c^{\theta} Q^{1+\theta} u_{x}^{2} d x d s \\
\leq & \frac{1}{4} \int_{0}^{t} \int_{0}^{1}(1+s) x^{-\frac{\theta+1}{\gamma}+\alpha}\left((c Q)^{\gamma}-\left(c Q_{\infty}\right)^{\gamma}\right)^{2} d x d s+C .
\end{aligned}
$$

Then from (5.6), (5.7), (5.8), we complete the proof of Corollary 5.3.

Lemma 5.4. Assume the conditions in Theorem 2.3 hold, we have

$$
\int_{0}^{1} x^{3-\frac{2 \theta+1}{\gamma}+\alpha}\left(\left((c Q)^{\theta}\right)_{x}-\left(\left(c Q_{\infty}\right)^{\theta}\right)_{x}\right)^{2} d x \leq \frac{C}{1+t}, \quad \forall t \geq 0
$$

and

$$
\int_{0}^{\infty} \int_{0}^{1}(1+t) x^{4-\frac{3 \theta+1}{\gamma}+\alpha}\left(\left((c Q)^{\theta}\right)_{x}-\left(\left(c Q_{\infty}\right)^{\theta}\right)_{x}\right)^{2} d x d t \leq C
$$

Proof. Multiplying (3.46) by $(1+s) x^{3-\frac{2 \theta+1}{\gamma}+\alpha}\left(\left((c Q)^{\theta}\right)_{x}-\left(\left(c Q_{\infty}\right)^{\theta}\right)_{x}+\theta \rho_{l} u\right)$, and integrating 
it on $[0,1] \times[0, t]$, we obtain

$$
\begin{aligned}
& \frac{1}{2}(1+t) \int_{0}^{1} x^{3-\frac{2 \theta+1}{\gamma}+\alpha}\left(\left((c Q)^{\theta}\right)_{x}-\left(\left(c Q_{\infty}\right)^{\theta}\right)_{x}+\theta \rho_{l} u\right)^{2} d x \\
& +\gamma \rho_{l} \int_{0}^{t} \int_{0}^{1}(1+s) x^{3-\frac{2 \theta+1}{\gamma}+\alpha}(c Q)^{\gamma-\theta}\left(\left((c Q)^{\theta}\right)_{x}-\left(\left(c Q_{\infty}\right)^{\theta}\right)_{x}+\theta \rho_{l} u\right)^{2} d x d s \\
= & \frac{1}{2} \int_{0}^{1} x^{3-\frac{2 \theta+1}{\gamma}+\alpha}\left(\left(\left(c Q_{0}\right)^{\theta}\right)_{x}-\left(\left(c Q_{\infty}\right)^{\theta}\right)_{x}+\theta \rho_{l} u_{0}\right)^{2} d x \\
& +\frac{1}{2} \int_{0}^{t} \int_{0}^{1} x^{3-\frac{2 \theta+1}{\gamma}+\alpha}\left(\left((c Q)^{\theta}\right)_{x}-\left(\left(c Q_{\infty}\right)^{\theta}\right)_{x}+\theta \rho_{l} u\right)^{2} d x d s \\
& +\int_{0}^{t} \int_{0}^{1}(1+s) x^{3-\frac{2 \theta+1}{\gamma}+\alpha}\left(\left((c Q)^{\theta}\right)_{x}-\left(\left(c Q_{\infty}\right)^{\theta}\right)_{x}+\theta \rho_{l} u\right) \theta \rho_{l}^{2} \gamma(c Q)^{\gamma-\theta} u d x d s \\
& +\theta \rho_{l} g \int_{0}^{t} \int_{0}^{1}(1+s) x^{3-\frac{2 \theta+1}{\gamma}+\alpha}\left(\left((c Q)^{\theta}\right)_{x}-\left(\left(c Q_{\infty}\right)^{\theta}\right)_{x}+\theta \rho_{l} u\right)\left(1-\frac{(c Q)^{\gamma-\theta}}{\left(c Q_{\infty}\right)^{\gamma-\theta}}\right) d x d s \\
& +\int_{0}^{t} \int_{0}^{1}(1+s) x^{3-\frac{2 \theta+1}{\gamma}+\alpha}\left(\left((c Q)^{\theta}\right)_{x}-\left(\left(c Q_{\infty}\right)^{\theta}\right)_{x}+\theta \rho_{l} u\right)\left(-\theta \rho_{l} h(Q) u|u|\right) d x d s \\
\leq & C+\frac{1}{2} \int_{0}^{t} \int_{0}^{1}(1+s) x^{3-\frac{2 \theta+1}{\gamma}+\alpha} \gamma \rho_{l}(c Q)^{\gamma-\theta}\left(\left((c Q)^{\theta}\right)_{x}-\left(\left(c Q_{\infty}\right)^{\theta}\right)_{x}+\theta \rho_{l} u\right)^{2} d x d s \\
& +C \int_{0}^{t} \int_{0}^{1}(1+s) x^{3-\frac{2 \theta+1}{\gamma}+\alpha}(c Q)^{\gamma-\theta} u^{2} d x d s \\
& +C \int_{0}^{t} \int_{0}^{1}(1+s) x^{3-\frac{2 \theta+1}{\gamma}+\alpha}(c Q)^{\theta-\gamma}\left(1-\frac{(c Q)^{\gamma-\theta}}{\left.\left(c Q_{\infty}\right)^{\gamma-\theta}\right)^{2} d x d s}\right. \\
& C \int_{0}^{t} \int_{0}^{1}(1+s) x^{3-\frac{2 \theta+1}{\gamma}+\alpha}(c Q)^{\theta-\gamma} h^{2}(Q) u^{4} d x d s, \\
&
\end{aligned}
$$

where we have used Lemma 3.9 and the assumption $\left(A_{2}\right)$.

By using Lemma 3.3, Corollaries 5.1 and 5.3, we get

$$
\begin{aligned}
& \int_{0}^{t} \int_{0}^{1}(1+s) x^{3-\frac{2 \theta+1}{\gamma}+\alpha}(c Q)^{\theta-\gamma}\left(1-\frac{(c Q)^{\gamma-\theta}}{(c Q)_{\infty}^{\gamma-\theta}}\right)^{2} d x d s \\
\leq & C \int_{0}^{t} \int_{0}^{1}(1+s) x^{-\frac{\theta+1}{\gamma}+\alpha}\left((c Q)^{\gamma}-(c Q)_{\infty}^{\gamma}\right)^{2} d x d s \leq C,
\end{aligned}
$$

and

$$
\int_{0}^{t} \int_{0}^{1}(1+s) x^{3-\frac{2 \theta+1}{\gamma}+\alpha}(c Q)^{\theta-\gamma} h^{2}(Q) u^{4} d x d s \leq C \int_{0}^{t} \int_{0}^{1}(1+s) u^{4} d x d s \leq C .
$$

From (5.11)-(5.13), we obtain (5.9) and (5.10) and the proof of Lemma 5.4 is complete.

Following the same ideas in Proposition 6.3 of [8], we can estimate the stabilization rate of $u(x, t)$ in the sense of $L^{\infty}$ norm under the conditions of Theorem 2.4.

Lemma 5.5. Assume the conditions in Theorem 2.3 hold, we have

$$
\int_{0}^{1} c^{\theta} Q^{1+\theta} u_{x}^{2} d x \leq \frac{C}{1+t}, \quad \forall t \geq 0
$$


and

$$
\int_{0}^{\infty} \int_{0}^{1}(1+t) u_{t}^{2} d x d t \leq C
$$

Proof. Multiplying $(\underbrace{}_{2.2})_{3}$ by $(1+s) u_{t}$, integrating the result equation over $[0,1] \times[0, t]$, integrating by parts, we get

$$
\begin{aligned}
& \int_{0}^{t} \int_{0}^{1}(1+s) u_{t}^{2} d x d s-\int_{0}^{t} \int_{0}^{1}(1+s)(c Q)^{\gamma} u_{t x} d x d s \\
= & -\frac{1}{3}(1+t) \int_{0}^{1} h(Q)|u|^{3} d x+\frac{1}{3} \int_{0}^{1} h\left(Q_{0}\right)\left|u_{0}\right|^{3} d x \\
& -\frac{1}{3} \rho_{l} \int_{0}^{t} \int_{0}^{1}(1+s) h^{\prime}(Q) Q^{2}|u|^{3} u_{x} d x d s+\frac{1}{3} \int_{0}^{t} \int_{0}^{1} h(Q)|u|^{3} d x d s \\
& -\int_{0}^{t} \int_{0}^{1}(1+s)\left(c Q_{\infty}\right)^{\gamma} u_{t x} d x d s-\int_{0}^{t} \int_{0}^{1}(1+s)\left(c^{\theta} Q^{1+\theta} u_{x}\right) u_{t x} d x d s .
\end{aligned}
$$

The last term on the right-hand side in (5.16) can be estimated as follows:

$$
\begin{aligned}
& -\int_{0}^{t} \int_{0}^{1}(1+s)\left(c^{\theta} Q^{1+\theta} u_{x}\right) u_{t x} d x d s \\
= & -\frac{1}{2}(1+t) \int_{0}^{1} c^{\theta} Q^{1+\theta} u_{x}^{2} d x+\frac{1}{2} \int_{0}^{1} c^{\theta} Q_{0}^{1+\theta} u_{0 x}^{2} d x \\
& +\frac{1}{2} \int_{0}^{t} \int_{0}^{1} c^{\theta} Q^{1+\theta} u_{x}^{2} d x d s-\frac{1}{2}(1+\theta) \rho_{l} \int_{0}^{t} \int_{0}^{1}(1+s) c^{\theta} Q^{2+\theta} u_{x}^{3} d x d s .
\end{aligned}
$$

Substituting (5.17) into (5.16), we get

$$
\begin{aligned}
& \int_{0}^{t} \int_{0}^{1}(1+s) u_{t}^{2} d x d s+\frac{1}{2}(1+t) \int_{0}^{1} c^{\theta} Q^{1+\theta} u_{x}^{2} d x+\frac{1}{3}(1+t) \int_{0}^{1} h(Q)|u|^{3} d x \\
= & \frac{1}{3} \int_{0}^{1} h\left(Q_{0}\right)\left|u_{0}\right|^{3} d x-\frac{1}{3} \rho_{l} \int_{0}^{t} \int_{0}^{1}(1+s) h^{\prime}(Q) Q^{2}|u|^{3} u_{x} d x d s+\frac{1}{3} \int_{0}^{t} \int_{0}^{1} h(Q)|u|^{3} d x d s \\
& +\frac{1}{2} \int_{0}^{1} c^{\theta} Q_{0}^{1+\theta} u_{0 x}^{2} d x+\frac{1}{2} \int_{0}^{t} \int_{0}^{1} c^{\theta} Q^{1+\theta} u_{x}^{2} d x d s-\frac{1}{2}(1+\theta) \rho_{l} \int_{0}^{t} \int_{0}^{1}(1+s) c^{\theta} Q^{2+\theta} u_{x}^{3} d x d s \\
& +\int_{0}^{t} \int_{0}^{1}(1+s)\left((c Q)^{\gamma}-\left(c Q_{\infty}\right)^{\gamma}\right) u_{t x} d x d s \\
= & \sum_{i=1}^{i=7} K_{i} .
\end{aligned}
$$

It is easy to see $K_{1}+K_{3}+K_{4}+K_{5} \leq C$ from the assumption $\left(A_{2}\right)$, Lemma 3.2 and Lemma 3.5.

$$
\begin{aligned}
K_{2} & \leq C \int_{0}^{t} \int_{0}^{1}(1+s) \frac{Q^{3}}{(1+Q)^{3}}|u|^{3}\left|u_{x}\right| d x d s \\
& \leq\left\|Q u_{x}\right\|_{L^{\infty}} \int_{0}^{t} \int_{0}^{1}(1+s)|u|^{3} d x d s \leq C,
\end{aligned}
$$

where we have used $\left\|Q u_{x}\right\|_{L^{\infty}} \leq C$ and Corollary 5.2. 
By using $\left\|Q u_{x}\right\|_{L^{\infty}} \leq C$ and Lemma 5.1, we have

$$
K_{6} \leq C\left\|Q u_{x}\right\|_{L^{\infty}} \int_{0}^{t} \int_{0}^{1}(1+s) c^{\theta} Q^{1+\theta} u_{x}^{2} d x d s \leq C .
$$

In order to complete the proof of Lemma 5.5, it suffices to estimate $K_{7}$ of the right-hand side in (5.18).

$$
\begin{aligned}
K_{7}= & (1+t) \int_{0}^{1}\left((c Q)^{\gamma}-\left(c Q_{\infty}\right)^{\gamma}\right) u_{x} d x-\int_{0}^{1}\left(\left(c Q_{0}\right)^{\gamma}-\left(c Q_{\infty}\right)^{\gamma}\right) u_{0 x} d x \\
& +\gamma \rho_{l} \int_{0}^{t} \int_{0}^{1}(1+s) c^{\gamma} Q^{1+\gamma} u_{x}^{2} d x d s-\int_{0}^{t} \int_{0}^{1}\left((c Q)^{\gamma}-\left(c Q_{\infty}\right)^{\gamma}\right) u_{x} d x d s \\
\leq & \frac{1}{4}(1+t) \int_{0}^{1} c^{\theta} Q^{1+\theta} u_{x}^{2} d x+C(1+t) \int_{0}^{1}\left((c Q)^{\gamma}-\left(c Q_{\infty}\right)^{\gamma}\right)^{2} c^{-\theta} Q^{-1-\theta} d x \\
& +\int_{0}^{1}\left(\left(c Q_{0}\right)^{\gamma}-\left(c Q_{\infty}\right)^{\gamma}\right)^{2} d x+\int_{0}^{1} u_{0 x}^{2} d x \\
& +\gamma \rho_{l} \int_{0}^{t} \int_{0}^{1}(c Q)^{\gamma-\theta}(1+s) c^{\theta} Q^{1+\theta} u_{x}^{2} d x d s+\int_{0}^{t} \int_{0}^{1}\left((c Q)^{\gamma}-\left(c Q_{\infty}\right)^{\gamma}\right)^{2} c^{-\theta} Q^{-1-\theta} d x d s \\
& +\int_{0}^{t} \int_{0}^{1} c^{\theta} Q^{1+\theta} u_{x}^{2} d x d s \\
\leq & C+C(1+t) \int_{0}^{1}\left((c Q)^{\gamma}-\left(c Q_{\infty}\right)^{\gamma}\right)^{2} c^{-\theta} Q^{-1-\theta} d x \\
& +\int_{0}^{t} \int_{0}^{1}\left((c Q)^{\gamma}-\left(c Q_{\infty}\right)^{\gamma}\right)^{2} c^{-\theta} Q^{-1-\theta} d x d s .
\end{aligned}
$$

Here, we have used Lemma 5.1, the assumption $\left(A_{2}\right)$ and Lemma 3.2. The rest two terms on the right-hand side of (5.21) can be estimated as follows:

$$
\begin{aligned}
C(1+t) \int_{0}^{1}\left((c Q)^{\gamma}-\left(c Q_{\infty}\right)^{\gamma}\right)^{2} c^{-\theta} Q^{-1-\theta} d x & \leq C(1+t) \int_{0}^{1}\left(c Q-c Q_{\infty}\right)^{2} x^{2-\frac{3+\theta}{\gamma}} d x \\
& \leq C(1+t) \int_{0}^{1}\left(c Q-c Q_{\infty}\right)^{2} x^{1-\frac{3}{\gamma}} d x \\
& \leq C,
\end{aligned}
$$

provided $\theta \leq \gamma-1$. And if we take $\beta=\gamma-\theta-1$ in Lemma 3.9, we have

$$
\begin{aligned}
& \int_{0}^{t} \int_{0}^{1}\left((c Q)^{\gamma}-\left(c Q_{\infty}\right)^{\gamma}\right)^{2} c^{-\theta} Q^{-1-\theta} d x d s \\
= & \int_{0}^{t} \int_{0}^{1} \frac{(c Q)^{\gamma}-\left(c Q_{\infty}\right)^{\gamma}}{(c Q)^{\beta}-\left(c Q_{\infty}\right)^{\beta}} c^{-\theta} Q^{-1-\theta}\left((c Q)^{\gamma}-\left(c Q_{\infty}\right)^{\gamma}\right)\left((c Q)^{\beta}-\left(c Q_{\infty}\right)^{\beta}\right) d x d s \\
\leq & C \int_{0}^{t} \int_{0}^{1}\left((c Q)^{\gamma}-\left(c Q_{\infty}\right)^{\gamma}\right)\left((c Q)^{\beta}-\left(c Q_{\infty}\right)^{\beta}\right) d x d s \leq C .
\end{aligned}
$$

Finally, from (5.18)-(5.23) we have

$$
\int_{0}^{t} \int_{0}^{1}(1+s) u_{t}^{2} d x d s+(1+t) \int_{0}^{1} c^{\theta} Q^{1+\theta} u_{x}^{2} d x+(1+t) \int_{0}^{1} h(Q)|u|^{2} d x \leq C .
$$

This proves Lemma 5.5. 


\section{Proof of Theorem 2.4.}

Choosing a positive number $m$ large enough, which is to be determined later, applying Hölder inequality, (2.6) and (5.9), we have by $(c Q)(0, t)=\left(c Q_{\infty}\right)(0, t)=0$

$$
\begin{aligned}
0 & \leq\left|(c Q)^{\theta}(x, t)-\left(c Q_{\infty}\right)^{\theta}(x, t)\right|^{m} \\
& \leq m \int_{0}^{x}\left|(c Q)^{\theta}-\left(c Q_{\infty}\right)^{\theta}\right|^{m-1}\left|\left((c Q)^{\theta}-\left(c Q_{\infty}\right)^{\theta}\right)_{x}\right| d y \\
& \leq m\left(\int_{0}^{1} x^{-\alpha+\frac{2 \theta+1}{\gamma}-3} \mid\left(c Q^{\theta}-\left.\left(c Q_{\infty}\right)^{\theta}\right|^{2 m-2} d x\right)^{\frac{1}{2}}\left(\int_{0}^{1} x^{3-\frac{2 \theta+1}{\gamma}+\alpha}\left((c Q)^{\theta}-\left(c Q_{\infty}\right)^{\theta}\right)_{x}^{2} d x\right)^{\frac{1}{2}}\right. \\
& \leq C(1+t)^{-\frac{1}{2}}\left(\int_{0}^{1} x^{-\alpha+\frac{2 \theta+1}{\gamma}-3}\left|(c Q)^{\theta}-\left(c Q_{\infty}\right)^{\theta}\right|^{2 m-2} d x\right)^{\frac{1}{2}} \\
& \leq C(1+t)^{-\frac{1}{2}}\left(\int_{0}^{1} x^{\frac{-\alpha \gamma+2 \theta+2-4 \gamma+\theta(2 m-2)}{\gamma}}\left(x^{1-\frac{3}{\gamma}}\left(c Q-c Q_{\infty}\right)^{2}\right) d x\right)^{\frac{1}{2}} .
\end{aligned}
$$

Taking $m=\frac{4 \gamma+\alpha \gamma-2}{2 \theta}$, then $2 \theta+2-4 \gamma+\theta(2 m-2)-\alpha \gamma=0$. It follows from (5.24) and (5.1) that (2.13) holds.

For the velocity function $u(x, t)$, (2.14) follows from (4.1) and (5.14) directly. This completes the proof of Theorem 2.4.

Acknowledgements: The authors were supported by the Natural Science Foundation of China \#11071093, the PhD specialized grant of the Ministry of Education of China \#20100144110001, and the Special Fund for Basic Scientific Research of Central Colleges \#CCNU10C01001.

\section{References}

[1] J.M. Delhaye, M. Giot, M.L. Riethmuller, Thermohydraulics of Two-Phase Systems for Industrial Design and Nuclear Engineering, Von Karman Institute, McGrawCHill, New York, 1981.

[2] Q. Duan, On the dynamics of Navier-Stokes equations for a shallow water model, J. Differential Equations, 250(2011), 2687-2714.

[3] S. Evje, K.-H. Karlsen, Global weak solutions for a viscous liquid-gas model with singular pressure law, Comm. Pure Appl. Anal., 8(2009), 1867-1894.

[4] S. Evje, K.K. Fjelde, On a rough AUSM scheme for a one dimensional two-phase model, Comput. \& Fluids, 32(2003), 1497-1530.

[5] H.-A. Friis, S. Evje, Global weak solutions for a gas-liquid model with external force and general pressure law, SIAM J. Appl. Math., 70(2011), 409-442.

[6] S. Evje, T. Flatten, On the wave structure of two-phase model, SIAM J. Appl. Math., $67(2006), 487-511$. 
[7] S. Evje, T. Flatten, H.-A. Friis, Global weak solutions for a viscous liquid-gas model with transition to single-phase gas flow and vacuum, Nonlinear Anal., TMA, 70(2009), 38643886 .

[8] T. Zhang, D.-Y. Fang, Global behavior of compressible Navier-Stokes equations with a degenerate viscosity coefficient, Arch. Rational Mech. Anal., 182(2006) 223-253.

[9] D.-Y. Fang, T. Zhang, Global behavior of spherically symmetric Navier-Stokes-Poisson system with degenerate viscosity coefficients, Arch. Rational Mech. Anal., 191(2009), 195243 .

[10] E. Feireisl, A. Novotny, H. Petzeltov, On the existence of globally defined weak solutions to the Navier-Stokes equations, J. Math. Fluid Mech., 3(2001), 358-392.

[11] Z.-H. Guo, C.-J. Zhu, Remarks on one-dimensional compressible Navier-Stokes equations with density-dependent viscosity and vacuum, Acta Math. Sin., 26(2010), 2015-2030.

[12] Z.-H. Guo, C.-J. Zhu, Global weak solutions and asymptotic behavior to 1D compressible Navier-Stokes equations with density-dependent viscosity and vacuum, J. Differential Equations, 248(2010), 2768-2799.

[13] Z.-H. Guo, Q.-S. Jiu, Z.-P. Xin, Spherically symmetric isentropic compressible flows with density-dependent viscosity coefficients, SIAM J. Math. Anal., 39(2008), 1402-1427.

[14] T. Nagasawa, On the asymptotic behavior of the one-dimensional motion of the polytropic ideal gas with stress-free condition, Quart. Appl. Math., 46(1988), 665-679.

[15] D. Hoff, Construction of solutions for compressible, isentropic Navier-Stokes equations in one space dimension with nonsmooth initial data, Proc. Roy. Soc. Edinburgh, Sect. A, 103(1986), 301-315.

[16] M. Okada, Free boundary value problems for the equation of one-dimensional motion of viscous gas, Japan J. Appl. Math., 6(1989), 161-177.

[17] I. Straskraba, A. Zlotnik, Global behavior of 1d-viscous compressible barotropic fluid with a free boundary and large data, J. Math. Fluid Mech., 5(2003), 119-143.

[18] T. Yang, H.-J. Zhao, A vacuum problem for the one-dimensional compressible NavierStokes equations with density-dependent viscosity, J. Differential Equations, 184(1)(2002), 163-184.

[19] T. Yang, C.-J. Zhu, Compressible Navier-Stokes equations with degenerate viscosity coefficient and vacuum, Comm. Math. Phys., 230(2002), 329-363.

[20] L. Yao, C.-J. Zhu, Free boundary value problem for a viscous two-phase model with massdependent viscosity, J. Differential Equations, 247(2009), 2705-2739.

[21] L. Yao, C.-J. Zhu, Existence and uniqueness of global weak solution to a two-phase flow model with vacuum, Math. Ann., 349(2011), 903-928.

[22] T. Yang, Z.-A. Yao, C.-J. Zhu, Compressible Navier-Stokes equations with densitydependent viscosity and vacuum, Comm. Partial Differential Equations, 26(2001), 965-981. 
[23] T. Yang, H.-J. Zhao, A vacuum problem for the one-dimensional compressible Navier-Stokes equations with density-dependent viscosity, J. Differential Equations, 184(2002), 163-184.

[24] T. Yang, C.-J. Zhu, Compressible Navier-Stokes equations with degenerate viscosity coefficient and vacuum, Comm. Math. Phys., 230(2002), 329-363.

[25] C.-J. Zhu, Asymptotic behavior of compressible Navier-Stokes equations with densitydependent viscosity and vacumm, Comm. Math. Phys., 293(2010), 279-299.

[26] C.-J. Zhu, R.-Z. Zi, Asymptotic behavior of solutions to 1D compressible Navier-Stokes equations with gravity and vacuum, Disc. Cont. Dyn. Sys., 30(2011), 1263-1283. 